\title{
Subleading contributions to the chiral three-nucleon force I: long-range terms
}

\author{
V. Bernard, ${ }^{1, *}$ E. Epelbaum, ${ }^{2,3, \dagger}$ H. Krebs, ${ }^{3, \text { 田 and Ulf-G. Meißner }}{ }^{3,2, \S}$ \\ ${ }^{1}$ Université Louis Pasteur, Laboratoire de Physique Théorique 3-5, rue de l'Université, F-67084 Strasbourg, France \\ ${ }^{2}$ Forschungszentrum Jülich, Institut für Kernphysik (Theorie), D-52425 Jülich, Germany \\ ${ }^{3}$ Universität Bonn, Helmholtz-Institut für Strahlen- und Kernphysik (Theorie), D-53115 Bonn, Germany
}

(Dated: October 27, 2018)

\begin{abstract}
We derive the long-range contributions to the tree-nucleon force at next-to-next-to-next-to-leading order in the chiral expansion. We give both momentum and coordinate space representations.
\end{abstract}

PACS numbers: $13.75 . \mathrm{Cs}, 21.30 .-\mathrm{x}$

\section{INTRODUCTION}

Three-nucleon forces (3NFs) are an indispensable ingredient in accurate few-nucleon and nuclear structure calculations. In particular, the three-nucleon system shows clear evidence for 3NFs, see the recent general introduction [1]. Chiral effective field theory is the appropriate tool to analyze nuclear forces. Precise two-nucleon potentials have been developed at next-to-next-to-next-to-leading order $\left(\mathrm{N}^{3} \mathrm{LO}\right)$ in the chiral expansion, see Refs. 2, 3]. 3NFs first appear at $\mathrm{N}^{2} \mathrm{LO}$ in the Weinberg counting scheme [4, 5] and have been analyzed and scrutinized in [6, 7, 8, 9, 10, 11]. There are various reasons to improve the theoretical precision of these 3NFs: 1) one should utilize the two- and three-nucleon forces at the same order in the expansion, 2) there are some outstanding discrepancies between theory and experiment like e.g the recently measured differential cross section in deuteron break-up at low energies [12] or the long-standing $A_{y}$ puzzle [13, 14] and 3) the theoretical uncertainty employing only the leading 3NFs quickly grows with increasing energy if one investigates e.g. nucleon-deuteron scattering or break-up. It is therefore timely and necessary to derive the chiral $3 \mathrm{NF}$ at $\mathrm{N}^{3} \mathrm{LO}$. In this work, we focus our attention on the long-range contributions at this order, which are free of unknown coupling constants. Here, we consider the chiral effective Lagrangian with pions and nucleons. The precise relation of the results presented here to an effective field theory including also spin- $3 / 2$ degrees of freedom will be the subject of a subsequent paper.

As we will show later, there are five different topologies contributing to the $3 \mathrm{NF}$ at $\mathrm{N}^{3} \mathrm{LO}$. From these, three topologies make up the long-range contribution, which is defined by not including any multi-nucleon contact interactions. This long-range part is given by two-pion exchange $(2 \pi)$ graphs, two-pion-one-pion exchange $(2 \pi-1 \pi)$ graphs and the socalled ring diagrams, where the pion loop connects all three nucleon lines. The first type of graphs has recently been considered based on the so-called infrared regularization in Ref. [15], we will compare our results to that work below. The spin-isospin structures originating from ring diagrams with one explicit delta intermediate state have been incorporated in the Illinois 3NF model [16]. Earlier, Coon and Friar [17] systematically constructed the 1/m (with $m$ the nucleon mass) corrections to the $2 \pi$ exchange $3 \mathrm{NF}$, and the so-called drift effects due to the boost of the two-nucleon force were worked out in [18]. We will compare to these works in our subsequent paper, where we discuss the shorter-ranged part of the $3 \mathrm{NF}$ at this order and the corresponding $1 / m$ corrections.

This manuscript is organized as follows. In Sect. [II we first write down the effective chiral Lagrangian and discuss the general structure of the $3 \mathrm{NF}$ at $\mathrm{N}^{3} \mathrm{LO}$. In the subsequent subsections IIA, IIB and IIC we give our results for the $2 \pi, 2 \pi-1 \pi$ and ring graphs, respectively, both in momentum and coordinate space representations. We end with

*Email: bernard@lpt6.u-strasbg.fr

†Email: e.epelbaum@fz-juelich.de

Email: hkrebs@itkp.uni-bonn.de

$\S$ Email: meissner@itkp.uni-bonn.de URL:www.itkp.uni-bonn.de/ meissner/ 
a summary and conclusions in Sect. III. In particular, we make some comments on the 3NF arising in an extension of this work with explicit delta degrees of freedom. The lengthy expressions for the momentum space representation of the ring diagrams are relegated to the appendix.

\section{LONG-RANGE CONTRIBUTIONS TO THE 3NF AT N ${ }^{3}$ LO}

The calculations performed in the following are based on the effective chiral Lagrangian for pions and nucleons. We employ here the heavy baryon formulation and display only the terms of relevance for our study:

$$
\begin{aligned}
\mathcal{L} & =\mathcal{L}^{\Delta=0}+\mathcal{L}^{\Delta=1}+\mathcal{L}^{\Delta=2}+\ldots \\
\mathcal{L}^{\Delta=0} & =\frac{F^{2}}{4}\left\langle\nabla^{\mu} U \nabla_{\mu} U^{\dagger}+\chi_{+}\right\rangle+\bar{N}\left(i v \cdot D+g_{A} u \cdot S\right) N+\ldots, \\
\mathcal{L}^{\Delta=1} & =\bar{N}\left(c_{1}\left\langle\chi_{+}\right\rangle+c_{2}(v \cdot u)^{2}+c_{3} u \cdot u+c_{4}\left[S^{\mu}, S^{\nu}\right] u_{\mu} u_{\nu}\right) N+\ldots, \\
\mathcal{L}^{\Delta=2} & =\bar{N}\left(d_{16} S \cdot u\left\langle\chi_{+}\right\rangle+i d_{18} S^{\mu}\left[D_{\mu}, \chi_{-}\right]+\tilde{d}_{28}\left(i\left\langle\chi_{+}\right\rangle v \cdot D+\text { h.c. }\right)+\ldots\right) N+\ldots,
\end{aligned}
$$

where the $c_{i}$ are low-energy constants and $N, v_{\mu}$ and $S_{\mu}$ denote the large component of the nucleon field, the nucleons four-velocity and the covariant spin vector, respectively. We use standard notation: $U(x)=u^{2}(x)$ collects the pion fields, $u_{\mu}=i\left(u^{\dagger} \partial_{\mu} u-u \partial_{\mu} u^{\dagger}\right), \chi_{+}=u^{\dagger} \chi u^{\dagger}+u \chi^{\dagger} u$ includes the explicit chiral symmetry breaking due to the finite light quark masses, $\langle\ldots\rangle$ denotes a trace in flavor space and $D_{\mu}$ is the chiral covariant derivative for the nucleon field. Notice further that the first terms in the expansion of $U(\boldsymbol{\pi})$ in powers of the pion fields read:

$$
U(\boldsymbol{\pi})=1+\frac{i}{F_{\pi}} \boldsymbol{\tau} \cdot \boldsymbol{\pi}-\frac{1}{2 F_{\pi}^{2}} \pi^{2}-\frac{i \alpha}{F_{\pi}^{3}}(\boldsymbol{\tau} \cdot \boldsymbol{\pi})^{3}+\frac{8 \alpha-1}{8 F_{\pi}^{4}} \boldsymbol{\pi}^{4}+\ldots,
$$

where $\boldsymbol{\tau}$ denote the Pauli isospin matrices and $\alpha$ is an arbitrary constant. For further notation and discussion, we refer to Ref. [19], a recent review is given in Ref. [20]. Following Weinberg [4, [5], the dimension $\Delta$ of the Lagrangian is defined via

$$
\Delta=d+\frac{1}{2} n-2
$$

where $d$ and $n$ are the number of derivatives or insertions of the pion mass $M_{\pi}$ and nucleon field operators, respectively. The pertinent low-energy constants (LECs) of the leading-order effective Lagrangian are the nucleon axial-vector coupling $g_{A}$ and the pion decay constant $F_{\pi}$. Notice that while all couplings and masses appearing in the effective Lagrangian should, strictly speaking, be taken at their SU(2) chiral limit values, to the accuracy we are working, we can use their pertinent physical values. In addition, we have the LECs $d_{16}, d_{18}$ and $\tilde{d}_{28}$ from the $\pi N$ Lagrangian at order $\Delta=2$. The ellipses in the brackets in the second line of Eq. (2.1) refer to terms proportional to the LECs $d_{1,2,3,5,14,15}$ and $\tilde{d}_{24,26,27,28,30}$ which generate $\pi \pi N N$ vertices [19] but do not contribute to the $3 \mathrm{NF}$ at $\mathrm{N}^{3} \mathrm{LO}$ as will be shown later. We also omit in Eq. (2.1) pion vertices with $\Delta=2$ and proportional to LECs $l_{3,4}$ which do not show up explicitly in the formulation based on renormalized pion fields at the considered order, see Ref. [21] for more details.

For a connected $N$-nucleon diagram with $L$ loops and $V_{i}$ vertices of dimension $\Delta_{i}$, the irreducible contribution ${ }^{1}$ to the scattering amplitude scales as $Q^{\nu}$, where $Q$ is a generic low-momentum scale associated with external nucleon three-momenta or $M_{\pi}$ and

$$
\nu=-4+2 N+2 L+\sum_{i} V_{i} \Delta_{i}
$$

Consequently, at $\mathrm{N}^{3} \mathrm{LO}$, which corresponds to $\nu=4$, one needs to take into account two classes of connected diagrams: tree diagrams with one insertion of the $\Delta=2$-interactions and one-loop graphs involving only lowest-order vertices with $\Delta=0$. Notice that it is not possible to draw $3 \mathrm{~N}$ diagrams with two insertions of $\Delta=1$-vertices at this order. We

\footnotetext{
1 This is the contribution which is not generated through iterations in the dynamical equation and which gives rise to the nuclear force.
} 


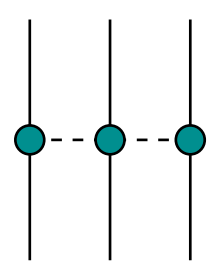

(a)

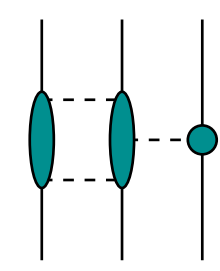

(b)

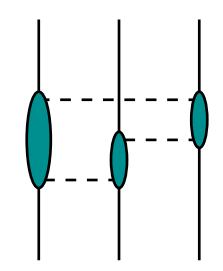

(c)

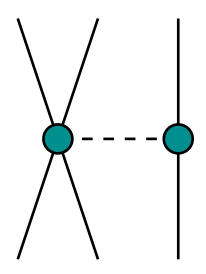

(d)

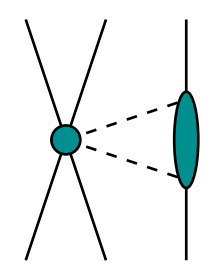

(e)

FIG. 1: Various topologies that appear in the $3 \mathrm{NF}$ at $\mathrm{N}^{3} \mathrm{LO}$. Solid and dashed lines represent nucleons and pions, respectively. Shaded blobs are the corresponding amplitudes. The long-range part of the $3 \mathrm{NF}$ considered in this paper consists of (a) $2 \pi$ exchange graphs, (b) $2 \pi-1 \pi$ diagrams and the so-called ring diagrams (c). The topologies (d) and (e) involve four-nucleon contact operators and are considered of shorter range.

further emphasize that similar to the case of the leading four-nucleon force considered in Refs. [22, 23], disconnected diagrams lead to vanishing contributions to the $3 \mathrm{NF}$ and will not be discussed in what follows.

The structure of the $3 \mathrm{NF}$ at $\mathrm{N}^{3} \mathrm{LO}$ is visualized in Fig. 1 and can be written as

$$
V_{3 \mathrm{~N}}^{(4)}=V_{2 \pi}^{(4)}+V_{2 \pi-1 \pi}^{(4)}+V_{\text {ring }}^{(4)}+V_{1 \pi-\text { cont }}^{(4)}+V_{2 \pi-\text { cont }}^{(4)}+V_{1 / m}^{(4)}
$$

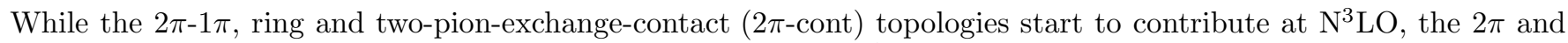
one-pion-exchange-contact ( $1 \pi$-cont) graphs already appear at $\mathrm{N}^{2} \mathrm{LO}$ yielding the following contributions to the $3 \mathrm{NF}$ [6, 8]:

$$
\begin{aligned}
V_{2 \pi}^{(3)} & =\frac{g_{A}^{2}}{8 F_{\pi}^{4}} \frac{\vec{\sigma}_{1} \cdot \vec{q}_{1} \vec{\sigma}_{3} \cdot \vec{q}_{3}}{\left[q_{1}^{2}+M_{\pi}^{2}\right]\left[q_{3}^{2}+M_{\pi}^{2}\right]}\left[\boldsymbol{\tau}_{1} \cdot \boldsymbol{\tau}_{3}\left(-4 c_{1} M_{\pi}^{2}+2 c_{3} \vec{q}_{1} \cdot \vec{q}_{3}\right)+c_{4} \boldsymbol{\tau}_{1} \times \boldsymbol{\tau}_{3} \cdot \boldsymbol{\tau}_{2} \vec{q}_{1} \times \vec{q}_{3} \cdot \vec{\sigma}_{2}\right], \\
V_{1 \pi-\text { cont }}^{(3)} & =-\frac{g_{A} D}{8 F_{\pi}^{2}} \frac{\vec{\sigma}_{3} \cdot \vec{q}_{3}}{q_{3}^{2}+M_{\pi}^{2}} \boldsymbol{\tau}_{1} \cdot \boldsymbol{\tau}_{3} \vec{\sigma}_{1} \cdot \vec{q}_{3},
\end{aligned}
$$

where the subscripts refer to the nucleon labels and $\vec{q}_{i}=\vec{p}_{i}{ }^{\prime}-\vec{p}_{i}$, with $\vec{p}_{i}{ }^{\prime}$ and $\vec{p}_{i}$ being the final and initial momenta of the nucleon $i$. Further, $q_{i} \equiv\left|\vec{q}_{i}\right|, \sigma_{i}$ denote the Pauli spin matrices and $D$ refers to the low-energy constant accompanying the leading $\pi N N N N$ vertex. Here and throughout this work, the results are always given for a particular choice of nucleon labels. The full expression for the $3 \mathrm{NF}$ results by taking into account all possible permutations of the nucleons ${ }^{2}$, i.e.:

$$
V_{3 \mathrm{~N}}^{\mathrm{full}}=V_{3 \mathrm{~N}}+\text { all permutations } .
$$

In this work, we focus on the long-range contributions $V_{2 \pi}^{(4)}, V_{2 \pi-1 \pi}^{(4)}$ and $V_{\text {ring }}^{(4)}$ resulting from diagrams (a), (b) and (c) in Fig. 11 respectively. The remaining terms resulting from graphs (d) and (e) in Fig. 1 and the relativistic $1 / m$-corrections will be discussed separately.

\section{A. Two-pion exchange topology}

The $2 \pi$ exchange diagrams at $\nu=4$ corresponding to topology (a) of Fig. 1 are depicted in Fig. 2. We do not show graphs involving tadpoles at pion lines which just renormalize the pion field and mass, see [21] for details. A close inspection of diagrams in Fig. 2 shows that most of them do not generate 3NFs. First, the contribution from graphs (12-17) involves an odd power of the loop momentum $\vec{l}$ to be integrated over and thus vanishes. Second, diagrams

\footnotetext{
${ }^{2}$ For three nucleons there are altogether 6 permutations.
} 


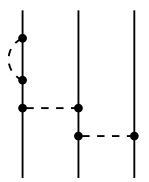

(1)

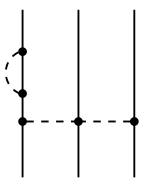

(8)

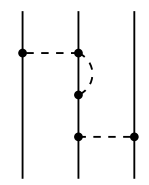

(15)

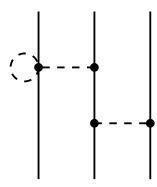

(22)

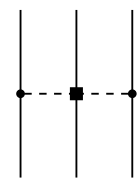

(29)

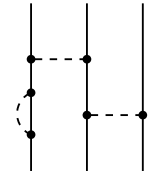

(2)

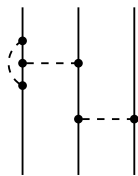

(3)

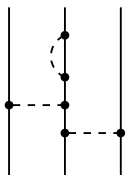

(4)

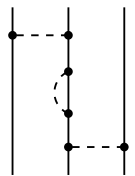

(5)

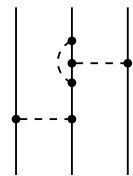

(6)

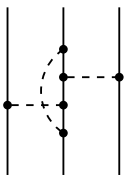

(7)

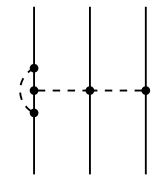

(9)

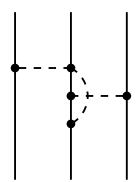

(16)

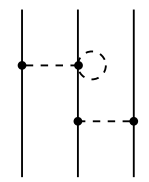

(23)

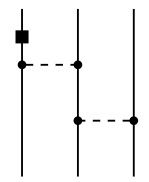

(30)

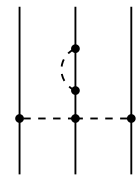

(10)

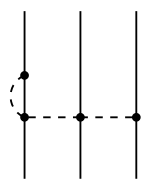

(17)

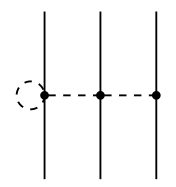

(24)

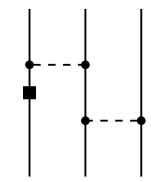

(31)

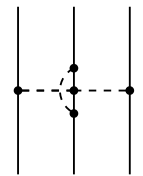

(11)

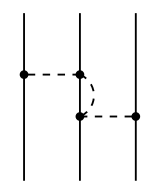

(18)

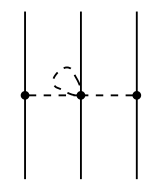

(25)

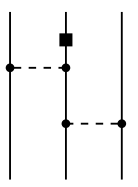

(32)

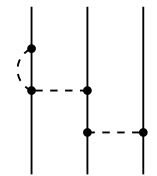

(12)

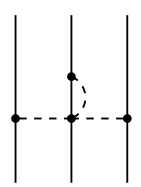

(19)

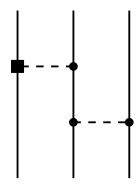

(26)

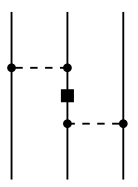

(33)

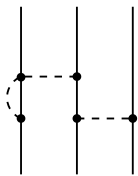

(13)

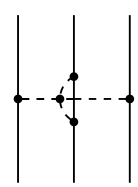

(20)

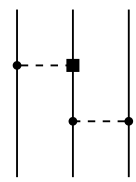

(27)

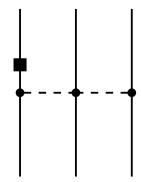

(34)

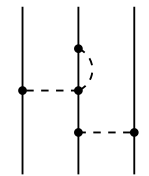

(14)

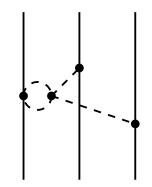

(21)

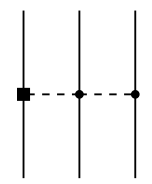

(28)

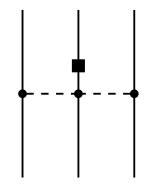

(35)

FIG. 2: Two-pion exchange $3 \mathrm{~N}$ diagrams at $\mathrm{N}^{3} \mathrm{LO}$. Solid dots (filled rectangles) denote vertices of dimension $\Delta_{i}=0\left(\Delta_{i}=2\right)$. Diagrams which result from the interchange of the nucleon lines and/or application of the time reversal operation are not shown. For remaining notation see Fig. 1 .

(1), (2), (4), (8), (10), (30-32), (34) and (35) just renormalize the external nucleon legs. Similarly, Feynman diagrams (3), (9), (22-24), (26-28) lead to renormalization of the leading pion-nucleon coupling without producing any new structures. All these contributions are taken into account by replacing the bare LECs in the leading $2 \pi$ exchange $3 \mathrm{~N}$ scattering amplitude by renormalized ones. This suggests that there are no $\mathrm{N}^{3} \mathrm{LO}$ corrections to the $3 \mathrm{NF}$ from these graphs since the $2 \pi$ exchange $3 \mathrm{~N}$ diagrams at order $\nu=2$ do not generate any nonvanishing $3 \mathrm{NF}$. Given the fact that nuclear potentials are, in general, not uniquely defined, the above argument based on the (on-shell) scattering amplitude should be taken with care. We have, however, verified that this is indeed the case by explicitly calculating the corresponding $3 \mathrm{NF}$ using the method of unitary transformation along the lines of Ref. 23]. From the remaining graphs in Fig. 2, diagram (11) does not contribute at the considered order due to the $1 / m$-suppression caused by the time derivative entering the Weinberg-Tomozawa vertex. ${ }^{3}$ For the same reason, diagram (25) also leads to a vanishing result at the order considered. Here, the time derivative acts either on the pions exchanged between two nucleons leading to a $1 / \mathrm{m}$-suppression or on the pion in the tadpole giving an odd power of the loop momentum $l^{0}$ to be

\footnotetext{
3 This graph does not involve reducible time-ordered topologies. Its contribution to the nuclear force is, therefore, most easily obtained using the Feynman graph technique. The $1 / m$-suppression from the time derivative entering the Weinberg-Tomozawa vertex follows then simply from the four-momentum conservation.
} 
integrated over. Further, it is easy to see that Feynman diagrams (18) and (21) do not contribute as well. Diagram (29) involves one insertion of the $\pi \pi N N$ vertices of dimension $\nu=2$. The relevant vertices are proportional to LECs $d_{1,2,3,5,14,15}$ and $\tilde{d}_{24,26,27,28,30}$. The corresponding $3 \mathrm{NF}$ is shifted to higher orders since all these vertices involve at least one time derivative, see [19] for explicit expressions. Last but not least, we also found that diagram (33) does not generate any $3 \mathrm{NF}$. Thus, we are left with diagrams (5-7), (19) and (20). The 3NF contribution from diagrams (5-7) can be evaluated straightforwardly using the expressions for the effective Hamilton operator from Ref. 23]. Diagrams (19) and (20) do not involve reducible topologies and can be evaluated using the Feynman graph technique. Notice that the individual contributions from graphs (19) and (20) in Fig. 2 and from diagram (20) in Fig. 3 depend on the arbitrary constant $\alpha$ which specifies the parametrization of the matrix $U$, see Eq. (2.2). Clearly, their sum is $\alpha$-independent.

We are now in the position to present our results. The expressions for diagrams (5-7) and (19) can be cast into the form of Eq. (2.6) leading only to shifts in the values of the LECs $c_{i}$

$$
c_{1} \rightarrow \bar{c}_{1}=c_{1}-\frac{g_{A}^{2} M_{\pi}}{64 \pi F_{\pi}^{2}}, \quad c_{3} \rightarrow \bar{c}_{3}=c_{3}+\frac{g_{A}^{4} M_{\pi}}{16 \pi F_{\pi}^{2}}, \quad c_{4} \rightarrow \bar{c}_{4}=c_{4}-\frac{g_{A}^{4} M_{\pi}}{16 \pi F_{\pi}^{2}},
$$

with $\delta c_{1}=-0.13 \mathrm{GeV}^{-1}$ and $\delta c_{3}=-\delta c_{4}=0.52 \mathrm{GeV}^{-1}$. These shifts are of the order of $20 \%$ to $30 \%$ of the corresponding LECs, and thus can not be neglected in precision studies of 3NFs. In contrast to this, the contribution from graph (20) takes a more complicated form compared to Eq. (2.6) and is given by

$$
\begin{aligned}
V_{2 \pi}^{(4)}= & \frac{g_{A}^{4}}{256 \pi F_{\pi}^{6}} \frac{\vec{\sigma}_{1} \cdot \vec{q}_{1} \vec{\sigma}_{3} \cdot \vec{q}_{3}}{\left[q_{1}^{2}+M_{\pi}^{2}\right]\left[q_{3}^{2}+M_{\pi}^{2}\right]}\left[\boldsymbol { \tau } _ { 1 } \cdot \boldsymbol { \tau } _ { 3 } \left(M_{\pi}\left(M_{\pi}^{2}+3 q_{1}^{2}+3 q_{3}^{2}+4 \vec{q}_{1} \cdot \vec{q}_{3}\right)+\left(2 M_{\pi}^{2}+q_{1}^{2}+q_{3}^{2}+2 \vec{q}_{1} \cdot \vec{q}_{3}\right)\right.\right. \\
& \left.\left.\times\left(3 M_{\pi}^{2}+3 q_{1}^{2}+3 q_{3}^{2}+4 \vec{q}_{1} \cdot \vec{q}_{3}\right) A\left(q_{2}\right)\right)-\boldsymbol{\tau}_{1} \times \boldsymbol{\tau}_{3} \cdot \boldsymbol{\tau}_{2} \vec{q}_{1} \times \vec{q}_{3} \cdot \vec{\sigma}_{2}\left(M_{\pi}+\left(4 M_{\pi}^{2}+q_{1}^{2}+q_{3}^{2}+2 \vec{q}_{1} \cdot \vec{q}_{3}\right) A\left(q_{2}\right)\right)\right] .
\end{aligned}
$$

Here, we have used dimensional regularization to evaluate the loop integrals. In this framework, the loop function $A(q)$ is given by:

$$
A(q)=\frac{1}{2 q} \arctan \frac{q}{2 M_{\pi}} .
$$

We further emphasize that the above expressions correspond to the choice $\alpha=0$. Notice also that some pieces in Eq. (2.9) can be brought into a form corresponding to the $2 \pi-1 \pi$ and $2 \pi$-cont topologies by canceling out the pion propagators with terms in the numerator. The $2 \pi$ exchange contributions arising from diagrams in Fig. 2 have also been considered recently by Ishikawa and Robilotta based on the infrared regularization [15]. We have verified that the long-range $2 \pi$ exchange contributions in Eqs. (2.6), (2.8) and (2.9) agree with the ones given in Ref. [15] provided the latter are expanded in powers of $1 / m$ and the leading terms are kept.

The coordinate space representation of the $2 \pi$ exchange $3 \mathrm{NF}$ can be obtained in a straightforward way. For the leading terms in the first line of Eq. (2.6) and the corrections in Eq. (2.8) one gets

$$
\begin{aligned}
V_{2 \pi}\left(\vec{r}_{12}, \vec{r}_{32}\right)= & \int \frac{d^{3} q_{1}}{(2 \pi)^{3}} \frac{d^{3} q_{3}}{(2 \pi)^{3}} e^{i \vec{q}_{1} \cdot \vec{r}_{12}} e^{i \vec{q}_{3} \cdot \vec{r}_{32}} V_{2 \pi}\left(\vec{q}_{1}, \vec{q}_{3}\right) \\
= & \frac{g_{A}^{2} M_{\pi}^{6}}{128 \pi^{2} F_{\pi}^{4}} \vec{\sigma}_{1} \cdot \vec{\nabla}_{12} \vec{\sigma}_{3} \cdot \vec{\nabla}_{32}\left[\boldsymbol{\tau}_{1} \cdot \boldsymbol{\tau}_{3}\left(4 \bar{c}_{1}+2 \bar{c}_{3} \vec{\nabla}_{12} \cdot \vec{\nabla}_{32}\right)+\bar{c}_{4} \boldsymbol{\tau}_{1} \times \boldsymbol{\tau}_{3} \cdot \boldsymbol{\tau}_{2} \vec{\nabla}_{12} \times \vec{\nabla}_{32} \cdot \vec{\sigma}_{2}\right] \\
& \times U_{1}\left(x_{12}\right) U_{1}\left(x_{32}\right),
\end{aligned}
$$

where $\vec{r}_{i j} \equiv \vec{r}_{i}-\vec{r}_{j}$ is the distance between the nucleons $i$ and $j$, the $\vec{x}_{i} \equiv M_{\pi} \vec{r}_{i}$ are dimensionless distances, the $\vec{\nabla}_{i}$ act on $\vec{x}_{i}$ and $x_{i j} \equiv\left|\vec{x}_{i j}\right|$. Further, the scalar function $U_{1}(x)$ is defined as:

$$
U_{1}(x)=\frac{4 \pi}{M_{\pi}} \int \frac{d^{3} q}{(2 \pi)^{3}} \frac{e^{i \vec{q} \cdot \vec{x} / M_{\pi}}}{q^{2}+M_{\pi}^{2}}=\frac{e^{-x}}{x} .
$$

Similarly, one obtains for the terms in Eq. (2.9) which do not involve the loop function $A\left(q_{2}\right)$ :

$$
\begin{aligned}
V_{2 \pi}\left(\vec{r}_{12}, \vec{r}_{32}\right)= & \frac{g_{A}^{4} M_{\pi}^{7}}{4096 \pi^{3} F_{\pi}^{6}} \vec{\sigma}_{1} \cdot \vec{\nabla}_{12} \vec{\sigma}_{3} \cdot \vec{\nabla}_{32}\left[\boldsymbol{\tau}_{1} \cdot \boldsymbol{\tau}_{3}\left(-1+3 \nabla_{12}^{2}+3 \nabla_{32}^{2}+4 \vec{\nabla}_{12} \cdot \vec{\nabla}_{32}\right)\right. \\
& \left.-\boldsymbol{\tau}_{1} \times \boldsymbol{\tau}_{3} \cdot \boldsymbol{\tau}_{2} \vec{\nabla}_{12} \times \vec{\nabla}_{32} \cdot \vec{\sigma}_{2}\right] U_{1}\left(x_{12}\right) U_{1}\left(x_{32}\right) .
\end{aligned}
$$




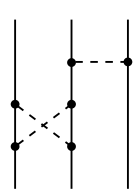

(1)

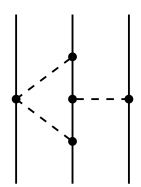

(8)

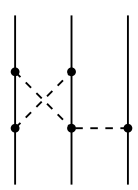

(15)

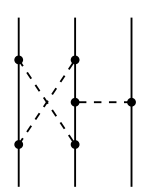

(2)

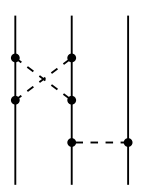

(3)

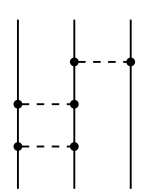

(4)

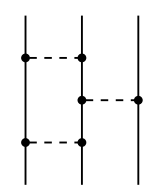

(5)

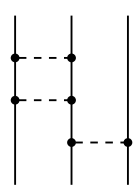

(6)

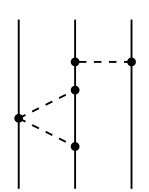

(7)

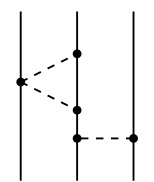

(9)

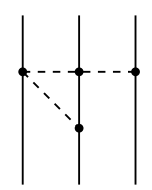

(16)

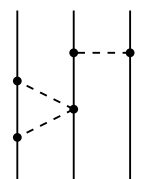

(10)

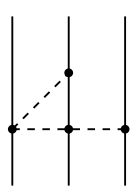

(17)

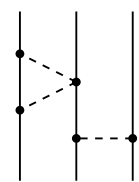

(11)

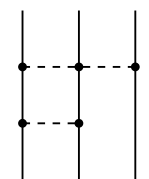

(12)

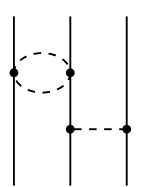

(18)

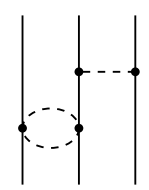

(19)

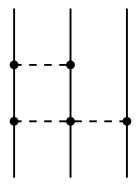

(13)

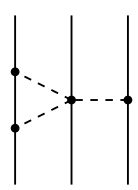

(20)

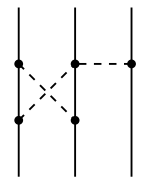

(14)

FIG. 3: $2 \pi-1 \pi$ diagrams at $\mathrm{N}^{3} \mathrm{LO}$. Graphs resulting from the interchange of the nucleon lines are not shown. For notation see Figs. 1, 2.

It should be understood that the obtained expressions are only valid in the region of space where the interparticle distances are large (i.e. larger than the inverse pion mass). The behavior of the potential at shorter distances is, in general, affected by the regularization procedure which is not considered in the present work.

To obtain the coordinate space representation for the terms in Eq. (2.9) which involve the loop function $A\left(q_{2}\right)$, it is more convenient to proceed in a different way in order to avoid a complicated angular integration:

$$
\begin{aligned}
V_{2 \pi}\left(\vec{r}_{12}, \vec{r}_{32}\right)= & \int \frac{d^{3} q_{1}}{(2 \pi)^{3}} \frac{d^{3} q_{2}}{(2 \pi)^{3}} \frac{d^{3} q_{3}}{(2 \pi)^{3}}(2 \pi)^{3} \delta^{3}\left(\vec{q}_{1}+\vec{q}_{2}+\vec{q}_{3}\right) e^{i \vec{q}_{1} \cdot \vec{r}_{1}} e^{i \vec{q}_{2} \cdot \vec{r}_{2}} e^{i \vec{q}_{3} \cdot \vec{r}_{3}} V_{2 \pi}\left(\vec{q}_{1}, \vec{q}_{2}, \vec{q}_{3}\right) \\
= & \int d^{3} r_{0} \int \frac{d^{3} q_{1}}{(2 \pi)^{3}} \frac{d^{3} q_{2}}{(2 \pi)^{3}} \frac{d^{3} q_{3}}{(2 \pi)^{3}} e^{i \vec{q}_{1} \cdot \vec{r}_{10}} e^{i \vec{q}_{2} \cdot \vec{r}_{20}} e^{i \vec{q}_{3} \cdot \vec{r}_{30}} V_{2 \pi}\left(\vec{q}_{1}, \vec{q}_{2}, \vec{q}_{3}\right) \\
= & -\frac{g_{A}^{4} M_{\pi}^{7}}{4096 \pi^{3} F_{\pi}^{6}} \vec{\sigma}_{1} \cdot \vec{\nabla}_{12} \vec{\sigma}_{3} \cdot \vec{\nabla}_{32}\left[\boldsymbol{\tau}_{1} \cdot \boldsymbol{\tau}_{3}\left(2-\nabla_{12}^{2}-\nabla_{32}^{2}-2 \vec{\nabla}_{12} \cdot \vec{\nabla}_{32}\right)\left(3-3 \nabla_{12}^{2}-3 \nabla_{32}^{2}-4 \vec{\nabla}_{12} \cdot \vec{\nabla}_{32}\right)\right. \\
& \left.+\boldsymbol{\tau}_{1} \times \boldsymbol{\tau}_{3} \cdot \boldsymbol{\tau}_{2} \vec{\nabla}_{12} \times \vec{\nabla}_{32} \cdot \vec{\sigma}_{2}\left(4-\nabla_{12}^{2}-\nabla_{32}^{2}-2 \vec{\nabla}_{12} \cdot \vec{\nabla}_{32}\right)\right] \\
& \times \frac{1}{4 \pi} \int d^{3} x U_{1}\left(\left|\vec{x}_{12}+\vec{x}\right|\right) W_{1}(x) U_{1}\left(\left|\vec{x}_{32}+\vec{x}\right|\right),
\end{aligned}
$$

where

$$
W_{1}(x)=\frac{4 \pi}{M_{\pi}^{2}} \int \frac{d^{3} q}{(2 \pi)^{3}} e^{i \vec{q} \cdot \vec{x} / M_{\pi}} A(q)=\frac{e^{-2 x}}{2 x^{2}}
$$

For various techniques to evaluate the integral in the last line of Eq. (2.14) the reader is referred to Ref. [15]. 


\section{B. Two-pion-one-pion exchange topology}

Consider now the $2 \pi-1 \pi 3 \mathrm{NF}$ arising from diagrams shown in Fig. 3 corresponding to topology (b) in Fig. 1 . They can be written in the form:

$$
\begin{aligned}
V_{2 \pi-1 \pi}= & \frac{\vec{\sigma}_{3} \cdot \vec{q}_{3}}{q_{3}^{2}+M_{\pi}^{2}}\left[\boldsymbol{\tau}_{1} \cdot \boldsymbol{\tau}_{3}\left(\vec{\sigma}_{2} \cdot \vec{q}_{1} \vec{q}_{1} \cdot \vec{q}_{3} F_{1}\left(q_{1}\right)+\vec{\sigma}_{2} \cdot \vec{q}_{1} F_{2}\left(q_{1}\right)+\vec{\sigma}_{2} \cdot \vec{q}_{3} F_{3}\left(q_{1}\right)\right)+\boldsymbol{\tau}_{2} \cdot \boldsymbol{\tau}_{3}\left(\vec{\sigma}_{1} \cdot \vec{q}_{1} \vec{q}_{1} \cdot \vec{q}_{3} F_{4}\left(q_{1}\right)\right.\right. \\
& \left.\left.+\vec{\sigma}_{1} \cdot \vec{q}_{3} F_{5}\left(q_{1}\right)+\vec{\sigma}_{2} \cdot \vec{q}_{1} F_{6}\left(q_{1}\right)+\vec{\sigma}_{2} \cdot \vec{q}_{3} F_{7}\left(q_{1}\right)\right)+\boldsymbol{\tau}_{1} \times \boldsymbol{\tau}_{2} \cdot \boldsymbol{\tau}_{3} \vec{\sigma}_{1} \times \vec{\sigma}_{2} \cdot \vec{q}_{1} F_{8}\left(q_{1}\right)\right]
\end{aligned}
$$

with $F_{1, \ldots, 8}\left(q_{1}\right)$ being scalar functions. Using the formal expressions for the effective Hamiltonian from Ref. [23] we obtain the following result for the first six graphs:

$$
\begin{aligned}
& F_{1}\left(q_{1}\right)=-\frac{g_{A}^{6}}{256 \pi F_{\pi}^{6}}\left[\frac{M_{\pi}}{4 M_{\pi}^{2}+q_{1}^{2}}+\frac{2 M_{\pi}}{q_{1}^{2}}-\frac{8 M_{\pi}^{2}+q_{1}^{2}}{q_{1}^{2}} A\left(q_{1}\right)\right] \\
& F_{3}\left(q_{1}\right)=-\frac{g_{A}^{6}}{256 \pi F_{\pi}^{6}}\left[3 M_{\pi}+\left(8 M_{\pi}^{2}+3 q_{1}^{2}\right) A\left(q_{1}\right)\right] \\
& F_{4}\left(q_{1}\right)=-\frac{1}{q_{1}^{2}} F_{5}\left(q_{1}\right)=-\frac{g_{A}^{6}}{128 \pi F_{\pi}^{6}} A\left(q_{1}\right) .
\end{aligned}
$$

Consider now diagrams (7-15) which involve one insertion of the Weinberg-Tomozawa vertex. The first three graphs only contribute to the functions $F_{1,3}\left(q_{1}\right)$ :

$$
\begin{aligned}
& F_{1}\left(q_{1}\right)=\frac{g_{A}^{4}}{256 \pi F_{\pi}^{6}}\left[\frac{M_{\pi}}{q_{1}^{2}}+\frac{q_{1}^{2}-4 M_{\pi}^{2}}{q_{1}^{2}} A\left(q_{1}\right)\right], \\
& F_{3}\left(q_{1}\right)=\frac{g_{A}^{4}}{256 \pi F_{\pi}^{6}}\left[M_{\pi}+\left(q_{1}^{2}+4 M_{\pi}^{2}\right) A\left(q_{1}\right)\right] .
\end{aligned}
$$

We find that diagrams (10) and (11) do not generate a 3NF while the contribution from graphs (12-15) has the form:

$$
F_{2}\left(q_{1}\right)=\frac{g_{A}^{4}}{128 \pi F_{\pi}^{6}}\left[M_{\pi}+\left(q_{1}^{2}+2 M_{\pi}^{2}\right) A\left(q_{1}\right)\right] .
$$

The next two diagrams (16) and (17) do not involve reducible topologies and can be dealt with using the Feynman graph technique. It is easy to see that their contribution to the scattering amplitude is suppressed by $1 / m$ due to the time derivative which enters the Weinberg-Tomozawa vertices. The next two diagrams (18) and (19) yield vanishing contributions to the $3 \mathrm{NF}$ for exactly the same reason as do the two-pion exchange diagrams $\propto g_{A}^{4}$ at order $\nu=2$. Next, the contribution from diagram (20) reads:

$$
\begin{aligned}
& F_{6}\left(q_{1}\right)=2 F_{7}\left(q_{1}\right)=\frac{g_{A}^{4}}{64 \pi F_{\pi}^{6}}\left[M_{\pi}+\left(q_{1}^{2}+2 M_{\pi}^{2}\right) A\left(q_{1}\right)\right] \\
& F_{8}\left(q_{1}\right)=-\frac{g_{A}^{4}}{512 \pi F_{\pi}^{6}}\left[M_{\pi}+\left(q_{1}^{2}+4 M_{\pi}^{2}\right) A\left(q_{1}\right)\right] .
\end{aligned}
$$

Finally, it is easy to see that the last Feynman diagram (21) in Fig. 3 yields a vanishing result at the order considered.

The coordinate space representation of the $2 \pi-1 \pi 3 \mathrm{NF}$ can be obtained straightforwardly employing the same Fouriertype integrations as in the the first line of Eq. (2.14). This leads to the following expression where short-range terms resulting from constant contributions to $F_{2,3,6,7,8}$, which are proportional to $M_{\pi}$, are not shown: :

$$
\begin{aligned}
V_{2 \pi-1 \pi}\left(\vec{r}_{12}, \vec{r}_{32}\right)= & \frac{g_{A}^{4} M_{\pi}^{7}}{8192 \pi^{3} F_{\pi}^{6}} \vec{\sigma}_{3} \cdot \vec{\nabla}_{32}\left(2 \boldsymbol { \tau } _ { 1 } \cdot \boldsymbol { \tau } _ { 3 } \left[\vec { \sigma } _ { 2 } \cdot \vec { \nabla } _ { 1 2 } \vec { \nabla } _ { 1 2 } \cdot \vec { \nabla } _ { 3 2 } \left(-2 g_{A}^{2} U_{1}\left(2 x_{12}\right)+\left(1+g_{A}^{2}\right) W_{1}\left(x_{12}\right)\right.\right.\right. \\
& \left.+\left(1-2 g_{A}^{2}\right) W_{3}\left(x_{12}\right)\right)-2 \vec{\sigma}_{2} \cdot \vec{\nabla}_{12}\left(2 W_{1}\left(x_{12}\right)+W_{2}\left(x_{12}\right)\right)-\vec{\sigma}_{2} \cdot \vec{\nabla}_{32}\left(4\left(1-2 g_{A}^{2}\right) W_{1}\left(x_{12}\right)\right. \\
& \left.\left.+\left(1-3 g_{A}^{2}\right) W_{2}\left(x_{12}\right)\right)\right]-4 \boldsymbol{\tau}_{2} \cdot \boldsymbol{\tau}_{3}\left[g_{A}^{2} \vec{\sigma}_{1} \cdot \vec{\nabla}_{12} \vec{\nabla}_{12} \cdot \vec{\nabla}_{32} W_{1}\left(x_{12}\right)+g_{A}^{2} \vec{\sigma}_{1} \cdot \vec{\nabla}_{32} W_{2}\left(x_{12}\right)\right. \\
& \left.+2 \vec{\sigma}_{2} \cdot \vec{\nabla}_{12}\left(2 W_{1}\left(x_{12}\right)+W_{2}\left(x_{12}\right)\right)+\vec{\sigma}_{2} \cdot \vec{\nabla}_{32}\left(2 W_{1}\left(x_{12}\right)+W_{2}\left(x_{12}\right)\right)\right] \\
& \left.+\boldsymbol{\tau}_{1} \times \boldsymbol{\tau}_{2} \cdot \boldsymbol{\tau}_{3} \vec{\sigma}_{1} \times \vec{\sigma}_{2} \cdot \vec{\nabla}_{12}\left(4 W_{1}\left(x_{12}\right)+W_{2}\left(x_{12}\right)\right)\right) U_{1}\left(x_{32}\right)
\end{aligned}
$$




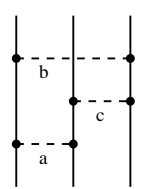

(1)

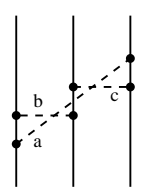

(2)

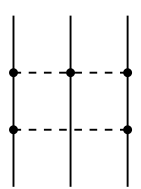

(3)

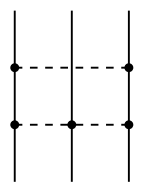

(4)

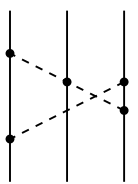

(5)

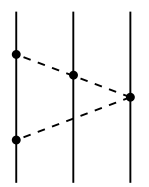

(6)

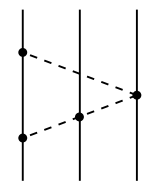

(7)

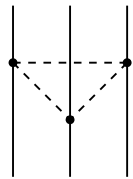

(8)

FIG. 4: Ring diagrams at $\mathrm{N}^{3} \mathrm{LO}$. Graphs resulting from the interchange of the nucleon lines are not shown. For notation see Figs. 1, 2,

with

$$
\begin{aligned}
& W_{2}(x)=-\nabla_{x}^{2} W_{1}(x)=-\frac{e^{-2 x}}{x^{4}}(1+2 x(1+x)) \\
& W_{3}(x)=\frac{4 \pi}{M_{\pi}^{2}} \int \frac{d^{3} q}{(2 \pi)^{3}} e^{i \vec{q} \cdot \vec{x} / M_{\pi}}\left[\frac{M_{\pi}}{q^{2}}-\frac{4 M_{\pi}^{2}}{q^{2}} A(q)\right]=2 E i(-2 x)+\frac{e^{-2 x}}{x}
\end{aligned}
$$

and

$$
E i(x) \equiv-\int_{-x}^{\infty} \frac{e^{-t} d t}{t}
$$

We emphasize again that the above expressions are only valid at large distances.

\section{Ring diagrams}

We now regard ring diagrams shown in Fig. 4 which correspond to topology (c) in Fig. 1. These are most cumbersome to evaluate. The contributions from the first two diagrams can be obtained using the expressions for the effective Hamilton operator given in Ref. [23]. This leads to the following structures:

$$
\begin{aligned}
& V_{\text {ring }}^{1}=M^{1}\left[\frac{4}{\omega_{a}^{3} \omega_{b} \omega_{c}}+\frac{4}{\omega_{a} \omega_{b}^{3} \omega_{c}}-\frac{4}{\omega_{a} \omega_{b} \omega_{c}^{3}}\right], \\
& V_{\text {ring }}^{2}=M^{2}\left[-\frac{4}{\omega_{a}^{3} \omega_{b} \omega_{c}}-\frac{4}{\omega_{a} \omega_{b}^{3} \omega_{c}}-\frac{4}{\omega_{a} \omega_{b} \omega_{c}^{3}}\right],
\end{aligned}
$$

where $M^{i}$ represents the spin, isospin and momentum structure which results from the vertices entering the diagram $i$ and $\omega$ denotes the pion free energy, $\omega \equiv \sqrt{q^{2}+M_{\pi}^{2}}$. Substituting the expressions for the vertices, the result can be written in the form

$$
V_{\text {ring }}=\left(\frac{g_{A}}{2 F_{\pi}}\right)^{6} \frac{1}{(2 \pi)^{3}} \int d^{3} l_{1} d^{3} l_{2} d^{3} l_{3} \delta^{3}\left(\vec{l}_{3}-\vec{l}_{2}-\vec{q}_{1}\right) \delta^{3}\left(\vec{l}_{2}-\vec{l}_{1}-\vec{q}_{3}\right) \frac{v}{\left[l_{1}^{2}+M_{\pi}^{2}\right]\left[l_{2}^{2}+M_{\pi}^{2}\right]^{2}\left[l_{3}^{2}+M_{\pi}^{2}\right]},
$$

with the numerator

$$
\begin{aligned}
v= & -8 \boldsymbol{\tau}_{1} \cdot \boldsymbol{\tau}_{2} \overrightarrow{l_{1}} \times \overrightarrow{l_{3}} \cdot \vec{\sigma}_{2} \overrightarrow{l_{1}} \times \overrightarrow{l_{2}} \cdot \vec{\sigma}_{3} \vec{l}_{2} \cdot \vec{l}_{3}-4 \boldsymbol{\tau}_{1} \cdot \boldsymbol{\tau}_{3} \vec{l}_{1} \cdot \vec{l}_{2} \vec{l}_{1} \cdot \vec{l}_{3} \vec{l}_{2} \cdot \vec{l}_{3}+2 \boldsymbol{\tau}_{1} \times \boldsymbol{\tau}_{2} \cdot \boldsymbol{\tau}_{3} \vec{l}_{1} \times \vec{l}_{3} \cdot \vec{\sigma}_{2} \vec{l}_{1} \cdot \vec{l}_{2} \vec{l}_{2} \cdot \vec{l}_{3} \\
& +6 \vec{l}_{2} \times \vec{l}_{3} \cdot \vec{\sigma}_{1} \vec{l}_{1} \times \vec{l}_{2} \cdot \vec{\sigma}_{3} \vec{l}_{1} \cdot \vec{l}_{3} .
\end{aligned}
$$

Carrying out the two trivial integrations over, say, $l_{1}$ and $l_{2}$ leads to the standard three-point function integrals. The latter can be evaluated but the resulting expressions are rather involved, see appendix A, It is more convenient to evaluate Eq. (2.25) in configuration space using again the same definition as in the the first line of Eq. (2.11). This leads to the following compact result:

$$
V_{\text {ring }}\left(\vec{r}_{12}, \vec{r}_{32}\right)=\left(\frac{g_{A}}{2 F_{\pi}}\right)^{6} \int \frac{d^{3} l_{1}}{(2 \pi)^{3}} \frac{d^{3} l_{2}}{(2 \pi)^{3}} \frac{d^{3} l_{3}}{(2 \pi)^{3}} e^{i \vec{l}_{1} \cdot \vec{r}_{23}} e^{i \vec{l}_{2} \cdot \vec{r}_{31}} e^{i \vec{l}_{3} \cdot \vec{r}_{12}} \frac{v}{\left[l_{1}^{2}+M_{\pi}^{2}\right]\left[l_{2}^{2}+M_{\pi}^{2}\right]^{2}\left[l_{3}^{2}+M_{\pi}^{2}\right]}
$$




$$
\begin{aligned}
= & -\frac{g_{A}^{6} M_{\pi}^{7}}{4096 \pi^{3} F_{\pi}^{6}}\left[-4 \boldsymbol{\tau}_{1} \cdot \boldsymbol{\tau}_{2} \vec{\nabla}_{23} \times \vec{\nabla}_{12} \cdot \vec{\sigma}_{2} \vec{\nabla}_{23} \times \vec{\nabla}_{31} \cdot \vec{\sigma}_{3} \vec{\nabla}_{31} \cdot \vec{\nabla}_{12}\right. \\
& -2 \boldsymbol{\tau}_{1} \cdot \boldsymbol{\tau}_{3} \vec{\nabla}_{23} \cdot \vec{\nabla}_{31} \vec{\nabla}_{23} \cdot \vec{\nabla}_{12} \vec{\nabla}_{31} \cdot \vec{\nabla}_{12}+\boldsymbol{\tau}_{1} \times \boldsymbol{\tau}_{2} \cdot \boldsymbol{\tau}_{3} \vec{\nabla}_{23} \times \vec{\nabla}_{12} \cdot \vec{\sigma}_{2} \vec{\nabla}_{23} \cdot \vec{\nabla}_{31} \vec{\nabla}_{31} \cdot \vec{\nabla}_{12} \\
& \left.+3 \vec{\nabla}_{31} \times \vec{\nabla}_{12} \cdot \vec{\sigma}_{1} \vec{\nabla}_{23} \times \vec{\nabla}_{31} \cdot \vec{\sigma}_{3} \vec{\nabla}_{23} \cdot \vec{\nabla}_{12}\right] U_{1}\left(x_{23}\right) U_{2}\left(x_{31}\right) U_{1}\left(x_{12}\right),
\end{aligned}
$$

where the derivatives should be evaluated as if the variables $\vec{x}_{12}, \vec{x}_{23}$ and $\vec{x}_{31}$ were independent ${ }^{4}$ and we have introduced

$$
U_{2}(x)=8 \pi M_{\pi} \int \frac{d^{3} q}{(2 \pi)^{3}} \frac{e^{i \vec{q} \cdot \vec{x} / M_{\pi}}}{\left[q^{2}+M_{\pi}^{2}\right]^{2}}=e^{-x} .
$$

Consider now diagrams (3-5) in Fig. 3 which involve one insertion of the Weinberg-Tomozawa vertex. The corresponding contribution to the $3 \mathrm{NF}$ can be evaluated in a similar way as described above and using the explicit expressions for the effective Hamilton operator from Ref. 23]. We find that graphs (3) and (4) lead to vanishing 3NF while the contribution from diagram $(5)$ reads:

$$
\begin{aligned}
V_{\text {ring }}\left(\vec{r}_{12}, \vec{r}_{32}\right)= & \frac{g_{A}^{4} M_{\pi}^{7}}{2048 \pi^{3} F_{\pi}^{6}}\left[2 \boldsymbol{\tau}_{1} \cdot \boldsymbol{\tau}_{2}\left(\vec{\nabla}_{23} \cdot \vec{\nabla}_{31} \vec{\nabla}_{31} \cdot \vec{\nabla}_{12}-\vec{\nabla}_{31} \times \vec{\nabla}_{12} \cdot \vec{\sigma}_{1} \vec{\nabla}_{23} \times \vec{\nabla}_{31} \cdot \vec{\sigma}_{3}\right)\right. \\
& \left.+\boldsymbol{\tau}_{1} \times \boldsymbol{\tau}_{2} \cdot \boldsymbol{\tau}_{3} \vec{\nabla}_{31} \times \vec{\nabla}_{12} \cdot \vec{\sigma}_{1} \vec{\nabla}_{23} \cdot \vec{\nabla}_{31}\right] U_{1}\left(x_{23}\right) U_{1}\left(x_{31}\right) U_{1}\left(x_{12}\right) .
\end{aligned}
$$

Finally, we found that the contributions from the last three diagrams in Fig. 3 are suppressed by the factor $1 / m$ and, therefore, need not to be taken into account at the order considered.

\section{SUMMARY AND CONCLUSIONS}

In this work, we have derived the long-range contributions of the $3 \mathrm{NF}$ in chiral effective field theory at $\mathrm{N}^{3} \mathrm{LO}$ in the chiral expansion. These results constitute the first systematic corrections to the leading $2 \pi$ exchange that appears at $\mathrm{N}^{2} \mathrm{LO}$ in the Weinberg counting. We have shown that there exists three different topologies that contribute to the long-range components of the chiral 3NF. While some of these simply renormalize the LECs of the leading order $2 \pi$ exchange topology, there are many new spin-isospin structures generated at $\mathrm{N}^{3} \mathrm{LO}$ from all three topologies. We have given the momentum and coordinate space representations of the various contributions. At this order, there is an arbitrariness in choosing the multi-pion interactions, cf. Eq. (2.2), which allows to shuffle strength between the various contributions. The sum of all diagrams contributing to the $3 \mathrm{NF}$ is, as demanded by field theory, independent of the choice of this parameter. To be precise, we have given our results for the special choice $\alpha=0$. It is important to stress that the corrections to the $3 \mathrm{NF}$ discussed here are free of any unknown low-energy constants and can be expressed entirely in terms of the nucleon axial-vector coupling $g_{A}$, the pion decay constant $F_{\pi}$ and the pion mass $M_{\pi}$. It should further be emphasized that the dimension-two LECs $c_{3}$ and $c_{4}$ which enter the leading 3NF and would also contribute at $\mathrm{N}^{4} \mathrm{LO}$ are larger than their natural values $c_{i} \sim g_{A} /\left(4 \pi F_{\pi}\right) \sim 1 \mathrm{GeV}^{-1}[20]$. This is understood in terms of $\Delta(1232) s$-channel and vector-meson $t$-channel excitations [24]. It is, therefore, expected that the theory with explicit spin-3/2 degrees of freedom leads to an improved convergence as compared to the pure pion-nucleon theory considered here. Of course, the theory with explicit deltas leads to many more structures and has also been much less developed phenomenologically. Still, it is important to confront the results obtained here with similarly accurate calculations in the delta-full theory. Work along these lines is in progress.

\section{Acknowledgments}

The work of E.E. and H.K. was supported in parts by funds provided from the Helmholtz Association to the young investigator group "Few-Nucleon Systems in Chiral Effective Field Theory" (grant VH-NG-222) and through the

\footnotetext{
${ }^{4}$ Clearly, the relative distances $\vec{r}_{12}, \vec{r}_{23}$ and $\vec{r}_{31}$ are related via $\vec{r}_{12}+\vec{r}_{23}+\vec{r}_{31}=0$
} 
virtual institute "Spin and strong QCD" (grant VH-VI-231). This work was further supported by the DFG (SFB/TR 16 "Subnuclear Structure of Matter") and by the EU Integrated Infrastructure Initiative Hadron Physics Project under contract number RII3-CT-2004-506078.

\section{APPENDIX A: EXPRESSIONS FOR RING DIAGRAMS IN MOMENTUM-SPACE}

In this appendix we give lengthy expressions for ring diagrams in Fig. 4 in momentum space. The contributions from diagrams (1) and (2) can be expressed as:

$$
\begin{aligned}
V_{\text {ring }} & =\vec{\sigma}_{1} \cdot \vec{\sigma}_{2} \boldsymbol{\tau}_{2} \cdot \boldsymbol{\tau}_{3} R_{1}+\vec{\sigma}_{1} \cdot \vec{q}_{1} \vec{\sigma}_{2} \cdot \vec{q}_{1} \boldsymbol{\tau}_{2} \cdot \boldsymbol{\tau}_{3} R_{2}+\vec{\sigma}_{1} \cdot \vec{q}_{1} \vec{\sigma}_{2} \cdot \vec{q}_{3} \boldsymbol{\tau}_{2} \cdot \boldsymbol{\tau}_{3} R_{3}+\vec{\sigma}_{1} \cdot \vec{q}_{3} \vec{\sigma}_{2} \cdot \vec{q}_{1} \boldsymbol{\tau}_{2} \cdot \boldsymbol{\tau}_{3} R_{4} \\
& +\vec{\sigma}_{1} \cdot \vec{q}_{3} \vec{\sigma}_{2} \cdot \vec{q}_{3} \boldsymbol{\tau}_{2} \cdot \boldsymbol{\tau}_{3} R_{5}+\boldsymbol{\tau}_{1} \cdot \boldsymbol{\tau}_{3} R_{6}+\vec{\sigma}_{1} \cdot \vec{q}_{1} \vec{\sigma}_{3} \cdot \vec{q}_{1} R_{7}+\vec{\sigma}_{1} \cdot \vec{q}_{1} \vec{\sigma}_{3} \cdot \vec{q}_{3} R_{8}+\vec{\sigma}_{1} \cdot \vec{q}_{3} \vec{\sigma}_{3} \cdot \vec{q}_{1} R_{9} \\
& +\vec{\sigma}_{1} \cdot \vec{\sigma}_{3} R_{10}+\vec{q}_{1} \cdot \vec{q}_{3} \times \vec{\sigma}_{2} \boldsymbol{\tau}_{1} \cdot \boldsymbol{\tau}_{2} \times \boldsymbol{\tau}_{3} R_{11} \cdot
\end{aligned}
$$

where the functions $R_{i} \equiv R_{i}\left(q_{1}, q_{3}, z\right)$ with $z=\hat{q}_{1} \cdot \hat{q}_{3}$ are defined as follows:

$$
\begin{aligned}
& R_{1}=\frac{\left(-1+z^{2}\right) g_{A}^{6} M_{\pi}\left(2 M_{\pi}^{2}+q_{3}^{2}\right)\left(q_{2}^{2} q_{3}+4 M_{\pi}^{2}\left(z q_{1}+q_{3}\right)\right)}{128 F^{6} \pi\left(4\left(-1+z^{2}\right) M_{\pi}^{2}-q_{2}^{2}\right)\left(4 M_{\pi}^{2} q_{3}+q_{3}^{3}\right)}-\frac{A\left(q_{2}\right) g_{A}^{6} q_{2}^{2}\left(2 M_{\pi}^{2}\left(q_{1}+z q_{3}\right)+z q_{3}\left(-q_{1}^{2}+q_{3}^{2}\right)\right)}{128 F^{6} \pi\left(-1+z^{2}\right) q_{1} q_{3}^{2}}- \\
& \frac{A\left(q_{3}\right) g_{A}^{6}\left(z q_{2}^{2}\left(z q_{1}-q_{3}\right) q_{3}+2 M_{\pi}^{2}\left(z\left(-2+z^{2}\right) q_{1}^{2}-\left(1+z^{2}\right) q_{1} q_{3}-z q_{3}^{2}\right)\right)}{128 F^{6} \pi\left(-1+z^{2}\right) q_{1} q_{3}}+ \\
& \frac{A\left(q_{1}\right) g_{A}^{6}\left(2 M_{\pi}^{2} q_{2}^{2}+q_{3}\left(-z q_{1}^{3}+\left(2-3 z^{2}\right) q_{1}^{2} q_{3}-z\left(-2+z^{2}\right) q_{1} q_{3}^{2}+q_{3}^{3}\right)\right)}{128 F^{6} \pi\left(-1+z^{2}\right) q_{3}^{2}}- \\
& \frac{I\left(4: 0,-q_{1}, q_{3} ; 0\right) g_{A}^{6} q_{2}^{2}}{32 F^{6}\left(-1+z^{2}\right)\left(4\left(-1+z^{2}\right) M_{\pi}^{2}-q_{2}^{2}\right) q_{3}}\left(8\left(-1+z^{2}\right) M_{\pi}^{4}\left(2 z q_{1}+\left(1+z^{2}\right) q_{3}\right)+q_{2}^{2} q_{3}\left(z^{2} q_{1}^{2}+\right.\right. \\
& \left.\left.z\left(-1+z^{2}\right) q_{1} q_{3}-q_{3}^{2}\right)+2 M_{\pi}^{2}\left(z\left(-2+z^{2}\right) q_{1}^{3}-\left(1+2 z^{2}\right) q_{1}^{2} q_{3}+3 z\left(-2+z^{2}\right) q_{1} q_{3}^{2}+\left(-3+2 z^{4}\right) q_{3}^{3}\right)\right) \\
& R_{2}=\frac{A\left(q_{2}\right) g_{A}^{6} q_{2}^{2}\left(-2 M_{\pi}^{2}\left(\left(1+z^{2}\right) q_{1}+2 z q_{3}\right)+z q_{3}\left(\left(1+z^{2}\right) q_{1}^{2}-2 q_{3}^{2}\right)\right)}{128 F^{6} \pi\left(-1+z^{2}\right)^{2} q_{1}^{3} q_{3}^{2}}+ \\
& \frac{A\left(q_{3}\right) g_{A}^{6}\left(M_{\pi}^{2}\left(2 z q_{1}^{2}+\left(1+3 z^{2}\right) q_{1} q_{3}+2 z q_{3}^{2}\right)+z q_{3}\left(-z q_{1}^{3}-z^{2} q_{1}^{2} q_{3}+z q_{1} q_{3}^{2}+q_{3}^{3}\right)\right)}{64 F^{6} \pi\left(-1+z^{2}\right)^{2} q_{1}^{3} q_{3}}+ \\
& \frac{A\left(q_{1}\right) g_{A}^{6}}{128 F^{6} \pi\left(-1+z^{2}\right)^{2} q_{1}^{2} q_{3}^{2}}\left(2 M_{\pi}^{2}\left(\left(1+z^{2}\right) q_{1}^{2}+z\left(3+z^{2}\right) q_{1} q_{3}+\left(1+z^{2}\right) q_{3}^{2}\right)+\right. \\
& \left.q_{3}\left(-\left(z+z^{3}\right) q_{1}^{3}+\left(2-5 z^{2}+z^{4}\right) q_{1}^{2} q_{3}+z\left(1+z^{2}\right) q_{1} q_{3}^{2}+\left(1+z^{2}\right) q_{3}^{3}\right)\right)- \\
& \frac{I\left(4: 0,-q_{1}, q_{3} ; 0\right) g_{A}^{6}}{32 F^{6}\left(-1+z^{2}\right)^{2} q_{1}^{2}\left(-4\left(-1+z^{2}\right) M_{\pi}^{2}+q_{2}^{2}\right) q_{3}}\left(q_{2}^{4} q_{3}\left(-2 z^{2} q_{1}^{2}+\left(1+z^{2}\right) q_{3}^{2}\right)-\right. \\
& 8(-1+z)(1+z) M_{\pi}^{4}\left(z\left(2+z^{2}\right) q_{1}^{3}+\left(1+2 z^{2}\right)^{2} q_{1}^{2} q_{3}+z\left(2+7 z^{2}\right) q_{1} q_{3}^{2}+\left(1+2 z^{2}\right) q_{3}^{3}\right)+ \\
& \left.2 M_{\pi}^{2} q_{2}^{2}\left(2 z q_{1}^{3}+\left(1-z^{2}+6 z^{4}\right) q_{1}^{2} q_{3}-2 z\left(-1-3 z^{2}+z^{4}\right) q_{1} q_{3}^{2}+\left(3+3 z^{2}-4 z^{4}\right) q_{3}^{3}\right)\right) \\
& +\frac{g_{A}^{6} M_{\pi}\left(2 M_{\pi}^{2}+q_{3}^{2}\right)\left(q_{2}^{2} q_{3}+4 M_{\pi}^{2}\left(z q_{1}+q_{3}\right)\right)}{128 F^{6} \pi q_{1}^{2}\left(4\left(-1+z^{2}\right) M_{\pi}^{2}-q_{2}^{2}\right)\left(4 M_{\pi}^{2} q_{3}+q_{3}^{3}\right)} \\
& R_{3}=-\frac{z A\left(q_{2}\right) g_{A}^{6} q_{2}^{2}\left(-4 M_{\pi}^{2}\left(q_{1}+z q_{3}\right)+q_{3}\left(2 z q_{1}^{2}+\left(-1+z^{2}\right) q_{1} q_{3}-2 z q_{3}^{2}\right)\right)}{128 F^{6} \pi\left(-1+z^{2}\right)^{2} q_{1}^{2} q_{3}^{3}}- \\
& \frac{z A\left(q_{3}\right) g_{A}^{6}}{128 F^{6} \pi\left(-1+z^{2}\right)^{2} q_{1}^{2} q_{3}^{2}}\left(M_{\pi}^{2}\left(-2 z\left(-3+z^{2}\right) q_{1}^{2}+4\left(1+z^{2}\right) q_{1} q_{3}+4 z q_{3}^{2}\right)+q_{3}\left(-\left(1+z^{2}\right) q_{1}^{3}-\right.\right. \\
& \left.\left.2 z^{3} q_{1}^{2} q_{3}+\left(1+z^{2}\right) q_{1} q_{3}^{2}+2 z q_{3}^{3}\right)\right)- \\
& \frac{z A\left(q_{1}\right) g_{A}^{6}\left(2 M_{\pi}^{2}\left(2 q_{1}^{2}+4 z q_{1} q_{3}+\left(1+z^{2}\right) q_{3}^{2}\right)+q_{3}\left(-2 z q_{1}^{3}+\left(1-3 z^{2}\right) q_{1}^{2} q_{3}+2 z q_{1} q_{3}^{2}+\left(1+z^{2}\right) q_{3}^{3}\right)\right)}{128 F^{6} \pi\left(-1+z^{2}\right)^{2} q_{1} q_{3}^{3}}- \\
& \frac{I\left(4: 0,-q_{1}, q_{3} ; 0\right) z g_{A}^{6}}{32 F^{6}\left(-1+z^{2}\right)^{2} q_{1}\left(-4\left(-1+z^{2}\right) M_{\pi}^{2}+q_{2}^{2}\right) q_{3}^{2}}\left(q_{2}^{4} q_{3}\left(\left(1+z^{2}\right) q_{1}^{2}+z\left(-1+z^{2}\right) q_{1} q_{3}-\left(1+z^{2}\right) q_{3}^{2}\right)+\right.
\end{aligned}
$$




$$
\begin{aligned}
& 8(-1+z)(1+z) M_{\pi}^{4}\left(3 z q_{1}^{3}+\left(-1+10 z^{2}\right) q_{1}^{2} q_{3}+3 z\left(1+2 z^{2}\right) q_{1} q_{3}^{2}+\left(1+2 z^{2}\right) q_{3}^{3}\right)+ \\
& \left.2 M_{\pi}^{2} q_{2}^{2}\left(z\left(-3+z^{2}\right) q_{1}^{3}+\left(3-9 z^{2}\right) q_{1}^{2} q_{3}-z\left(5+z^{2}\right) q_{1} q_{3}^{2}+\left(-3-3 z^{2}+4 z^{4}\right) q_{3}^{3}\right)\right)+ \\
& \frac{z g_{A}^{6} M_{\pi}\left(2 M_{\pi}^{2}+q_{3}^{2}\right)\left(q_{2}^{2} q_{3}+4 M_{\pi}^{2}\left(z q_{1}+q_{3}\right)\right)}{128 F^{6} \pi q_{1}\left(-4\left(-1+z^{2}\right) M_{\pi}^{2}+q_{2}^{2}\right) q_{3}^{2}\left(4 M_{\pi}^{2}+q_{3}^{2}\right)} \\
& R_{4}=\frac{A\left(q_{2}\right) g_{A}^{6} q_{2}^{2}\left(-2 z^{2} q_{1}^{2} q_{3}+\left(1+z^{2}\right) q_{3}^{3}+2 M_{\pi}^{2}\left(2 z q_{1}+\left(1+z^{2}\right) q_{3}\right)\right)}{128 F^{6} \pi\left(-1+z^{2}\right)^{2} q_{1}^{2} q_{3}^{3}}+ \\
& \frac{A\left(q_{1}\right) g_{A}^{6}\left(-2 M_{\pi}^{2}\left(2 z q_{1}^{2}+\left(1+3 z^{2}\right) q_{1} q_{3}+2 z q_{3}^{2}\right)+q_{3}\left(2 z^{2} q_{1}^{3}+2 z^{3} q_{1}^{2} q_{3}+\left(1-4 z^{2}+z^{4}\right) q_{1} q_{3}^{2}-2 z q_{3}^{3}\right)\right)}{128 F^{6} \pi\left(-1+z^{2}\right)^{2} q_{1} q_{3}^{3}}- \\
& \frac{A\left(q_{3}\right) g_{A}^{6}}{128 F^{6} \pi\left(-1+z^{2}\right)^{2} q_{1}^{2} q_{3}^{2}}\left(2 M_{\pi}^{2}\left(-z^{2}\left(-3+z^{2}\right) q_{1}^{2}+z\left(3+z^{2}\right) q_{1} q_{3}+\left(1+z^{2}\right) q_{3}^{2}\right)+\right. \\
& \left.q_{3}\left(-\left(z+z^{3}\right) q_{1}^{3}-\left(1-z^{2}+2 z^{4}\right) q_{1}^{2} q_{3}+z\left(1+z^{2}\right) q_{1} q_{3}^{2}+\left(1+z^{2}\right) q_{3}^{3}\right)\right)- \\
& \frac{I\left(4: 0,-q_{1}, q_{3} ; 0\right) g_{A}^{6}}{32 F^{6}\left(-1+z^{2}\right)^{2} q_{1}\left(-4\left(-1+z^{2}\right) M_{\pi}^{2}+q_{2}^{2}\right) q_{3}^{2}}\left(q_{2}^{4} q_{3}\left(\left(z+z^{3}\right) q_{1}^{2}+\left(-1+z^{2}\right)^{2} q_{1} q_{3}-2 z q_{3}^{2}\right)+\right. \\
& 8(-1+z)(1+z) M_{\pi}^{4}\left(3 z^{2} q_{1}^{3}+9 z^{3} q_{1}^{2} q_{3}+\left(-2+9 z^{2}+2 z^{4}\right) q_{1} q_{3}^{2}+z\left(2+z^{2}\right) q_{3}^{3}\right)+ \\
& \left.2 M_{\pi}^{2} q_{2}^{2}\left(z^{2}\left(-3+z^{2}\right) q_{1}^{3}+\left(2 z-8 z^{3}\right) q_{1}^{2} q_{3}+\left(4+5 z^{2}\left(-3+z^{2}\right)\right) q_{1} q_{3}^{2}+2 z\left(-3+z^{2}+z^{4}\right) q_{3}^{3}\right)\right)+ \\
& \frac{z g_{A}^{6} M_{\pi}\left(2 M_{\pi}^{2}+q_{3}^{2}\right)\left(q_{2}^{2} q_{3}+4 M_{\pi}^{2}\left(z q_{1}+q_{3}\right)\right)}{128 F^{6} \pi q_{1}\left(-4\left(-1+z^{2}\right) M_{\pi}^{2}+q_{2}^{2}\right) q_{3}^{2}\left(4 M_{\pi}^{2}+q_{3}^{2}\right)} \\
& R_{5}=\frac{A\left(q_{2}\right) g_{A}^{6} q_{2}^{2}\left(-4 M_{\pi}^{2}\left(q_{1}+z q_{3}\right)+q_{3}\left(2 z q_{1}^{2}+\left(-1+z^{2}\right) q_{1} q_{3}-2 z q_{3}^{2}\right)\right)}{128 F^{6} \pi\left(-1+z^{2}\right)^{2} q_{1} q_{3}^{4}}- \\
& \frac{A\left(q_{3}\right) g_{A}^{6}}{128 F^{6} \pi\left(-1+z^{2}\right)^{2} q_{1} q_{3}^{3}}\left(2 M_{\pi}^{2}\left(z\left(-3+z^{2}\right) q_{1}^{2}-2\left(1+z^{2}\right) q_{1} q_{3}-2 z q_{3}^{2}\right)+q_{3}\left(\left(1+z^{2}\right) q_{1}^{3}+2 z^{3} q_{1}^{2} q_{3}-\right.\right. \\
& \left.\left.\left(1+z^{2}\right) q_{1} q_{3}^{2}-2 z q_{3}^{3}\right)\right)+ \\
& \frac{A\left(q_{1}\right) g_{A}^{6}\left(2 M_{\pi}^{2}\left(2 q_{1}^{2}+4 z q_{1} q_{3}+\left(1+z^{2}\right) q_{3}^{2}\right)+q_{3}\left(-2 z q_{1}^{3}+\left(1-3 z^{2}\right) q_{1}^{2} q_{3}+2 z q_{1} q_{3}^{2}+\left(1+z^{2}\right) q_{3}^{3}\right)\right)}{128 F^{6} \pi\left(-1+z^{2}\right)^{2} q_{3}^{4}}+ \\
& \frac{I\left(4: 0,-q_{1}, q_{3} ; 0\right) g_{A}^{6}}{32 F^{6}\left(-1+z^{2}\right)^{2}\left(-4\left(-1+z^{2}\right) M_{\pi}^{2}+q_{2}^{2}\right) q_{3}^{3}}\left(q_{2}^{4} q_{3}\left(\left(1+z^{2}\right) q_{1}^{2}+z\left(-1+z^{2}\right) q_{1} q_{3}-\left(1+z^{2}\right) q_{3}^{2}\right)+\right. \\
& 8(-1+z)(1+z) M_{\pi}^{4}\left(3 z q_{1}^{3}+\left(-1+10 z^{2}\right) q_{1}^{2} q_{3}+3 z\left(1+2 z^{2}\right) q_{1} q_{3}^{2}+\left(1+2 z^{2}\right) q_{3}^{3}\right)+ \\
& \left.2 M_{\pi}^{2} q_{2}^{2}\left(z\left(-3+z^{2}\right) q_{1}^{3}+\left(3-9 z^{2}\right) q_{1}^{2} q_{3}-z\left(5+z^{2}\right) q_{1} q_{3}^{2}+\left(-3-3 z^{2}+4 z^{4}\right) q_{3}^{3}\right)\right)- \\
& \frac{g_{A}^{6} M_{\pi}\left(2 M_{\pi}^{2}+q_{3}^{2}\right)\left(q_{2}^{2} q_{3}+4 M_{\pi}^{2}\left(z q_{1}+q_{3}\right)\right)}{128 F^{6} \pi\left(-4\left(-1+z^{2}\right) M_{\pi}^{2}+q_{2}^{2}\right) q_{3}^{3}\left(4 M_{\pi}^{2}+q_{3}^{2}\right)}, \\
& R_{6}=\frac{A\left(q_{2}\right) g_{A}^{6}\left(2 M_{\pi}^{2}+q_{2}^{2}\right)}{128 F^{6} \pi}+\frac{A\left(q_{1}\right) g_{A}^{6}\left(2 z\left(M_{\pi}^{2}+q_{1}^{2}\right) q_{3}+q_{1}\left(8 M_{\pi}^{2}+3 q_{1}^{2}+q_{3}^{2}\right)\right)}{128 F^{6} \pi q_{1}}+ \\
& \frac{A\left(q_{3}\right) g_{A}^{6}\left(2 z q_{1}\left(M_{\pi}^{2}+q_{3}^{2}\right)+q_{3}\left(8 M_{\pi}^{2}+q_{1}^{2}+3 q_{3}^{2}\right)\right)}{128 F^{6} \pi q_{3}}- \\
& \frac{g_{A}^{6} M_{\pi}}{128 F^{6} \pi q_{1}\left(4 M_{\pi}^{2}+q_{1}^{2}\right)\left(4\left(-1+z^{2}\right) M_{\pi}^{2}-q_{2}^{2}\right) q_{3}\left(4 M_{\pi}^{2}+q_{3}^{2}\right)}\left(\left(5+z^{2}\right) q_{1}^{3} q_{2}^{2} q_{3}^{3}+8 M_{\pi}^{6}\left(z\left(-3+4 z^{2}\right) q_{1}^{2}+\right.\right. \\
& \left.2\left(19-18 z^{2}\right) q_{1} q_{3}+z\left(-3+4 z^{2}\right) q_{3}^{2}\right)+2 M_{\pi}^{4}\left(4 z\left(-1+z^{2}\right) q_{1}^{4}+\left(77-36 z^{2}\right) q_{1}^{3} q_{3}+2 z\left(33+8 z^{2}\right) q_{1}^{2} q_{3}^{2}+\right. \\
& \left.\left(77-36 z^{2}\right) q_{1} q_{3}^{3}+4 z\left(-1+z^{2}\right) q_{3}^{4}\right)+2 M_{\pi}^{2} q_{1} q_{3}\left(\left(10+z^{2}\right) q_{1}^{4}+2 z\left(9+2 z^{2}\right) q_{1}^{3} q_{3}+\left(29-7 z^{2}\right) q_{1}^{2} q_{3}^{2}+\right. \\
& \left.\left.2 z\left(9+2 z^{2}\right) q_{1} q_{3}^{3}+\left(10+z^{2}\right) q_{3}^{4}\right)\right)- \\
& \frac{I\left(4: 0,-q_{1}, q_{3} ; 0\right) g_{A}^{6}\left(2 M_{\pi}^{2}+q_{2}^{2}\right)}{32 F^{6} q_{1}\left(-4\left(-1+z^{2}\right) M_{\pi}^{2}+q_{2}^{2}\right) q_{3}}\left(q_{1} q_{2}^{2} q_{3}\left(q_{1}^{2}+z q_{1} q_{3}+q_{3}^{2}\right)+4 M_{\pi}^{4}\left(z q_{1}^{2}-2\left(-2+z^{2}\right) q_{1} q_{3}+z q_{3}^{2}\right)+\right. \\
& \left.2 M_{\pi}^{2}\left(4 q_{1} q_{3}\left(q_{1}^{2}+q_{3}^{2}\right)+z\left(q_{1}^{4}+6 q_{1}^{2} q_{3}^{2}+q_{3}^{4}\right)\right)\right),
\end{aligned}
$$




$$
\begin{aligned}
& R_{7}=\frac{3 g_{A}^{6} M_{\pi}\left(2 M_{\pi}^{2}+q_{2}^{2}\right)}{256 F^{6} \pi q_{1}^{2}\left(-4\left(-1+z^{2}\right) M_{\pi}^{2}+q_{2}^{2}\right)}-\frac{3 A\left(q_{3}\right) g_{A}^{6}\left(2 M_{\pi}^{2}+q_{2}^{2}\right)\left(\left(1+z^{2}\right) q_{1}+2 z q_{3}\right)}{256 F^{6} \pi\left(-1+z^{2}\right)^{2} q_{1}^{3}}- \\
& \frac{3 A\left(q_{1}\right) g_{A}^{6}\left(2 M_{\pi}^{2}+q_{2}^{2}\right)\left(2 z q_{1}+\left(1+z^{2}\right) q_{3}\right)}{256 F^{6} \pi\left(-1+z^{2}\right)^{2} q_{1}^{2} q_{3}}+\frac{3 A\left(q_{2}\right) g_{A}^{6}\left(2 M_{\pi}^{2}+q_{2}^{2}\right)\left(2 z q_{1}^{2}+\left(1+3 z^{2}\right) q_{1} q_{3}+2 z q_{3}^{2}\right)}{256 F^{6} \pi\left(-1+z^{2}\right)^{2} q_{1}^{3} q_{3}}+ \\
& \frac{3 I\left(4: 0,-q_{1}, q_{3} ; 0\right) g_{A}^{6}\left(2 M_{\pi}^{2}+q_{2}^{2}\right)}{64 F^{6}\left(-1+z^{2}\right)^{2} q_{1}^{2}\left(4\left(-1+z^{2}\right) M_{\pi}^{2}-q_{2}^{2}\right)}\left(-q_{2}^{2}\left(\left(1+z^{2}\right) q_{1}^{2}+z\left(3+z^{2}\right) q_{1} q_{3}+\left(1+z^{2}\right) q_{3}^{2}\right)+\right. \\
& \left.4\left(-1+z^{2}\right) M_{\pi}^{2}\left(\left(1+2 z^{2}\right) q_{1}^{2}+2 z\left(2+z^{2}\right) q_{1} q_{3}+\left(1+2 z^{2}\right) q_{3}^{2}\right)\right), \\
& R_{8}=-\frac{3 z g_{A}^{6} M_{\pi}\left(2 M_{\pi}^{2}+q_{2}^{2}\right)}{256 F^{6} \pi q_{1}\left(-4\left(-1+z^{2}\right) M_{\pi}^{2}+q_{2}^{2}\right) q_{3}}+\frac{3 z A\left(q_{3}\right) g_{A}^{6}\left(2 M_{\pi}^{2}+q_{2}^{2}\right)\left(\left(1+z^{2}\right) q_{1}+2 z q_{3}\right)}{256 F^{6} \pi\left(-1+z^{2}\right)^{2} q_{1}^{2} q_{3}}+ \\
& \frac{3 z A\left(q_{1}\right) g_{A}^{6}\left(2 M_{\pi}^{2}+q_{2}^{2}\right)\left(2 z q_{1}+\left(1+z^{2}\right) q_{3}\right)}{256 F^{6} \pi\left(-1+z^{2}\right)^{2} q_{1} q_{3}^{2}}-\frac{3 z A\left(q_{2}\right) g_{A}^{6}\left(2 M_{\pi}^{2}+q_{2}^{2}\right)\left(2 z q_{1}^{2}+\left(1+3 z^{2}\right) q_{1} q_{3}+2 z q_{3}^{2}\right)}{256 F^{6} \pi\left(-1+z^{2}\right)^{2} q_{1}^{2} q_{3}^{2}}- \\
& \frac{3 I\left(4: 0,-q_{1}, q_{3} ; 0\right) z g_{A}^{6}\left(2 M_{\pi}^{2}+q_{2}^{2}\right)}{64 F^{6}\left(-1+z^{2}\right)^{2} q_{1}\left(4\left(-1+z^{2}\right) M_{\pi}^{2}-q_{2}^{2}\right) q_{3}}\left(-q_{2}^{2}\left(\left(1+z^{2}\right) q_{1}^{2}+z\left(3+z^{2}\right) q_{1} q_{3}+\left(1+z^{2}\right) q_{3}^{2}\right)+\right. \\
& \left.4\left(-1+z^{2}\right) M_{\pi}^{2}\left(\left(1+2 z^{2}\right) q_{1}^{2}+2 z\left(2+z^{2}\right) q_{1} q_{3}+\left(1+2 z^{2}\right) q_{3}^{2}\right)\right), \\
& R_{9}=-\frac{3 A\left(q_{2}\right) g_{A}^{6}\left(2 M_{\pi}^{2}+q_{2}^{2}\right)\left(\left(1+z^{2}\right) q_{1}^{2}+z\left(3+z^{2}\right) q_{1} q_{3}+\left(1+z^{2}\right) q_{3}^{2}\right)}{256 F^{6} \pi\left(-1+z^{2}\right)^{2} q_{1}^{2} q_{3}^{2}}+ \\
& \frac{3 A\left(q_{1}\right) g_{A}^{6}\left(\left(1+z^{2}\right) q_{1}^{3}+2 z\left(2+z^{2}\right) q_{1}^{2} q_{3}-z^{2}\left(-7+z^{2}\right) q_{1} q_{3}^{2}+2 z q_{3}^{3}+2 M_{\pi}^{2}\left(\left(1+z^{2}\right) q_{1}+2 z q_{3}\right)\right)}{256 F^{6} \pi\left(-1+z^{2}\right)^{2} q_{1} q_{3}^{2}}+ \\
& \frac{3 A\left(q_{3}\right) g_{A}^{6}\left(2 z q_{1}^{3}-z^{2}\left(-7+z^{2}\right) q_{1}^{2} q_{3}+2 z\left(2+z^{2}\right) q_{1} q_{3}^{2}+\left(1+z^{2}\right) q_{3}^{3}+2 M_{\pi}^{2}\left(2 z q_{1}+\left(1+z^{2}\right) q_{3}\right)\right)}{256 F^{6} \pi\left(-1+z^{2}\right)^{2} q_{1}^{2} q_{3}}+ \\
& \frac{3 I\left(4: 0,-q_{1}, q_{3} ; 0\right) z g_{A}^{6}\left(2 M_{\pi}^{2}+q_{2}^{2}\right)}{64 F^{6}\left(-1+z^{2}\right)^{2} q_{1}\left(-4\left(-1+z^{2}\right) M_{\pi}^{2}+q_{2}^{2}\right) q_{3}}\left(q_{2}^{2}\left(-2 q_{1}^{2}+z\left(-5+z^{2}\right) q_{1} q_{3}-2 q_{3}^{2}\right)+\right. \\
& \left.4\left(-1+z^{2}\right) M_{\pi}^{2}\left(\left(2+z^{2}\right) q_{1}^{2}+6 z q_{1} q_{3}+\left(2+z^{2}\right) q_{3}^{2}\right)\right)-\frac{3 z g_{A}^{6} M_{\pi}\left(2 M_{\pi}^{2}+q_{2}^{2}\right)}{256 F^{6} \pi q_{1}\left(-4\left(-1+z^{2}\right) M_{\pi}^{2}+q_{2}^{2}\right) q_{3}}, \\
& R_{10}=\frac{3\left(-1+z^{2}\right) g_{A}^{6} M_{\pi}\left(2 M_{\pi}^{2}+q_{2}^{2}\right)}{256 F^{6} \pi\left(-4\left(-1+z^{2}\right) M_{\pi}^{2}+q_{2}^{2}\right)}+\frac{3 A\left(q_{2}\right) g_{A}^{6}\left(2 M_{\pi}^{2}+q_{2}^{2}\right)\left(z q_{1}+q_{3}\right)\left(q_{1}+z q_{3}\right)}{256 F^{6} \pi\left(-1+z^{2}\right) q_{1} q_{3}}- \\
& \frac{3 A\left(q_{1}\right) g_{A}^{6}\left(z q_{1}^{3}+\left(1+2 z^{2}\right) q_{1}^{2} q_{3}-z\left(-4+z^{2}\right) q_{1} q_{3}^{2}+q_{3}^{3}+2 M_{\pi}^{2}\left(z q_{1}+q_{3}\right)\right)}{256 F^{6} \pi\left(-1+z^{2}\right) q_{3}}- \\
& \frac{3 A\left(q_{3}\right) g_{A}^{6}\left(q_{1}^{3}-z\left(-4+z^{2}\right) q_{1}^{2} q_{3}+\left(1+2 z^{2}\right) q_{1} q_{3}^{2}+z q_{3}^{3}+2 M_{\pi}^{2}\left(q_{1}+z q_{3}\right)\right)}{256 F^{6} \pi\left(-1+z^{2}\right) q_{1}}+ \\
& \frac{3 I\left(4: 0,-q_{1}, q_{3} ; 0\right) g_{A}^{6}\left(2 M_{\pi}^{2}+q_{2}^{2}\right)}{64 F^{6}\left(-1+z^{2}\right)\left(4\left(-1+z^{2}\right) M_{\pi}^{2}-q_{2}^{2}\right)}\left(-q_{2}^{2}\left(q_{1}^{2}-z\left(-3+z^{2}\right) q_{1} q_{3}+q_{3}^{2}\right)+\right. \\
& \left.4\left(-1+z^{2}\right) M_{\pi}^{2}\left(\left(1+z^{2}\right) q_{1}^{2}+4 z q_{1} q_{3}+\left(1+z^{2}\right) q_{3}^{2}\right)\right), \\
& R_{11}=-\frac{A\left(q_{2}\right) g_{A}^{6} q_{2}^{2}\left(4 M_{\pi}^{2}+q_{1}^{2}+q_{3}^{2}\right)}{256 F^{6} \pi\left(-1+z^{2}\right) q_{1}^{2} q_{3}^{2}}+ \\
& \frac{A\left(q_{3}\right) g_{A}^{6}\left(2 M_{\pi}^{2}\left(\left(-1+z^{2}\right) q_{1}^{2}+2 z q_{1} q_{3}+2 q_{3}^{2}\right)+q_{3}\left(z q_{1}^{3}+\left(-1+2 z^{2}\right) q_{1}^{2} q_{3}+z q_{1} q_{3}^{2}+q_{3}^{3}\right)\right)}{256 F^{6} \pi\left(-1+z^{2}\right) q_{1}^{2} q_{3}^{2}}+ \\
& \frac{A\left(q_{1}\right) g_{A}^{6}\left(2 M_{\pi}^{2}\left(2 q_{1}^{2}+2 z q_{1} q_{3}+\left(-1+z^{2}\right) q_{3}^{2}\right)+q_{1}\left(q_{1}^{3}+z q_{1}^{2} q_{3}+\left(-1+2 z^{2}\right) q_{1} q_{3}^{2}+z q_{3}^{3}\right)\right)}{256 F^{6} \pi\left(-1+z^{2}\right) q_{1}^{2} q_{3}^{2}}- \\
& \frac{I\left(4: 0,-q_{1}, q_{3} ; 0\right) g_{A}^{6} q_{2}^{2}}{\left(64 F^{6}\left(-1+z^{2}\right) q_{1}^{2}\left(-4\left(-1+z^{2}\right) M_{\pi}^{2}+q_{2}^{2}\right) q_{3}^{2}\right)}\left(-\left(2 M_{\pi}^{2}+q_{1}^{2}\right)\left(2 M_{\pi}^{2}+q_{3}^{2}\right)\left(4 M_{\pi}^{2}+q_{1}^{2}+q_{3}^{2}\right)+\right. \\
& 2 z^{3} q_{1} q_{3}\left(-4 M_{\pi}^{4}+q_{1}^{2} q_{3}^{2}\right)+z^{2}\left(4 M_{\pi}^{2}+q_{1}^{2}+q_{3}^{2}\right)\left(4 M_{\pi}^{4}+3 q_{1}^{2} q_{3}^{2}+2 M_{\pi}^{2}\left(q_{1}^{2}+q_{3}^{2}\right)\right)+ \\
& \left.z q_{1} q_{3}\left(8 M_{\pi}^{4}+q_{1}^{4}+q_{3}^{4}+4 M_{\pi}^{2}\left(q_{1}^{2}+q_{3}^{2}\right)\right)\right)-
\end{aligned}
$$




$$
\begin{aligned}
& \frac{g_{A}^{6} M_{\pi}}{256 F^{6} \pi q_{1}^{2}\left(4 M_{\pi}^{2}+q_{1}^{2}\right)\left(-4\left(-1+z^{2}\right) M_{\pi}^{2}+q_{2}^{2}\right) q_{3}^{2}\left(4 M_{\pi}^{2}+q_{3}^{2}\right)}\left(-2 z^{2}\left(4 M_{\pi}^{4} q_{1}^{2}\left(4 M_{\pi}^{2}+q_{1}^{2}\right)+\right.\right. \\
& \left.4 M_{\pi}^{2}\left(2 M_{\pi}^{2}+q_{1}^{2}\right)^{2} q_{3}^{2}+\left(2 M_{\pi}^{2}+q_{1}^{2}\right)^{2} q_{3}^{4}\right)+2 M_{\pi}^{2}\left(4 M_{\pi}^{2}+q_{1}^{2}+q_{3}^{2}\right)\left(q_{1}^{2} q_{3}^{2}+2 M_{\pi}^{2}\left(q_{1}^{2}+q_{3}^{2}\right)\right)- \\
& \left.z q_{1} q_{3}\left(32 M_{\pi}^{6}+12 M_{\pi}^{4}\left(q_{1}^{2}+q_{3}^{2}\right)+q_{1}^{2} q_{3}^{2}\left(q_{1}^{2}+q_{3}^{2}\right)+2 M_{\pi}^{2}\left(q_{1}^{4}+4 q_{1}^{2} q_{3}^{2}+q_{3}^{4}\right)\right)\right) .
\end{aligned}
$$

In the above expressions, $q_{1}$ and $q_{3}$ are always to be understood as the magnitudes of the corresponding three-momenta (except in the arguments of the function $I), q_{1} \equiv\left|\vec{q}_{1}\right|, q_{3} \equiv\left|\vec{q}_{3}\right|$. Further, the function $I\left(d: p_{1}, p_{2}, p_{3} ; p_{4}\right)$ refers to the scalar loop integral

$$
I\left(d: p_{1}, p_{2}, p_{3} ; p_{4}\right)=\frac{1}{i} \int \frac{d^{d} l}{(2 \pi)^{d}} \frac{1}{\left(l+p_{1}\right)^{2}-M_{\pi}^{2}+i \epsilon} \frac{1}{\left(l+p_{2}\right)^{2}-M_{\pi}^{2}+i \epsilon} \frac{1}{\left(l+p_{3}\right)^{2}-M_{\pi}^{2}+i \epsilon} \frac{1}{v \cdot\left(l+p_{4}\right)+i \epsilon} .
$$

In a general case, this function depends on the four-momenta $p_{i}$. For the case $p_{i}^{0}=0$ which we are interested in, it can be expressed in terms of the three-point function in Euclidean space $J\left(d: \vec{p}_{1}, \vec{p}_{2}, \vec{p}_{3}\right)$

$$
J\left(d: \vec{p}_{1}, \vec{p}_{2}, \vec{p}_{3}\right)=\int \frac{d^{d} l}{(2 \pi)^{d}} \frac{1}{\left(\vec{l}+\vec{p}_{1}\right)^{2}+M_{\pi}^{2}} \frac{1}{\left(\vec{l}+\vec{p}_{2}\right)^{2}+M_{\pi}^{2}} \frac{1}{\left(\vec{l}+\vec{p}_{3}\right)^{2}+M_{\pi}^{2}} .
$$

In particular, the function $I\left(4: 0,-q_{1}, q_{3} ; 0\right)$ which enters the expressions for $R_{i}$ can be written as

$$
I\left(4: 0,-q_{1}, q_{3} ; 0\right)=\frac{1}{2} J\left(3: \overrightarrow{0},-\vec{q}_{1}, \vec{q}_{3}\right) .
$$

For diagram (5), we obtain the following representation:

$$
\begin{aligned}
V_{\text {ring }} & =\boldsymbol{\tau}_{1} \cdot \boldsymbol{\tau}_{2} S_{1}+\vec{\sigma}_{1} \cdot \vec{q}_{1} \vec{\sigma}_{3} \cdot \vec{q}_{1} \boldsymbol{\tau}_{1} \cdot \boldsymbol{\tau}_{2} S_{2}+\vec{\sigma}_{1} \cdot \vec{q}_{3} \vec{\sigma}_{3} \cdot \vec{q}_{1} \boldsymbol{\tau}_{1} \cdot \boldsymbol{\tau}_{2} S_{3}+\vec{\sigma}_{1} \cdot \vec{q}_{1} \vec{\sigma}_{3} \cdot \vec{q}_{3} \boldsymbol{\tau}_{1} \cdot \boldsymbol{\tau}_{2} S_{4} \\
& +\vec{\sigma}_{1} \cdot \vec{q}_{3} \vec{\sigma}_{3} \cdot \vec{q}_{3} \boldsymbol{\tau}_{1} \cdot \boldsymbol{\tau}_{2} S_{5}+\vec{\sigma}_{1} \cdot \vec{\sigma}_{3} \boldsymbol{\tau}_{1} \cdot \boldsymbol{\tau}_{2} S_{6}+\vec{q}_{1} \cdot \vec{q}_{3} \times \vec{\sigma}_{1} \boldsymbol{\tau}_{1} \cdot \boldsymbol{\tau}_{2} \times \boldsymbol{\tau}_{3} S_{7},
\end{aligned}
$$

where the functions $S_{i} \equiv S_{i}\left(q_{1}, q_{3}, z\right)$ are given by

$$
\begin{aligned}
S_{1}= & -\frac{A\left(q_{1}\right) g_{A}^{4}\left(2 M_{\pi}^{2}+q_{1}^{2}\right)}{128 F^{6} \pi}-\frac{A\left(q_{2}\right) g_{A}^{4}\left(4 M_{\pi}^{2}+q_{1}^{2}+z q_{1} q_{3}+q_{3}^{2}\right)}{128 F^{6} \pi}-\frac{A\left(q_{3}\right) g_{A}^{4}\left(2 M_{\pi}^{2}+q_{3}^{2}\right)}{128 F^{6} \pi}+ \\
& \frac{I\left(4: 0,-q_{1}, q_{3} ; 0\right) g_{A}^{4}\left(2 M_{\pi}^{2}+q_{1}^{2}\right)\left(2 M_{\pi}^{2}+q_{3}^{2}\right)}{32 F^{6}}-\frac{g_{A}^{4} M_{\pi}}{64 F^{6} \pi} \\
S_{2}= & -\frac{A\left(q_{1}\right) g_{A}^{4}\left(\left(1+z^{2}\right) q_{1}+2 z q_{3}\right)}{128 F^{6} \pi\left(-1+z^{2}\right)^{2} q_{1}}-\frac{A\left(q_{3}\right) g_{A}^{4} q_{3}\left(2 z q_{1}+\left(1+z^{2}\right) q_{3}\right)}{128 F^{6} \pi\left(-1+z^{2}\right)^{2} q_{1}^{2}}+ \\
& \frac{I\left(4: 0,-q_{1}, q_{3} ; 0\right) g_{A}^{4} q_{3}\left(-4 z\left(-1+z^{2}\right) M_{\pi}^{2}+2 z q_{1}^{2}+\left(1+3 z^{2}\right) q_{1} q_{3}+2 z q_{3}^{2}\right)}{32 F^{6}\left(-1+z^{2}\right)^{2} q_{1}}+ \\
& \frac{A\left(q_{2}\right) g_{A}^{4}\left(\left(1+z^{2}\right) q_{1}^{2}+z\left(3+z^{2}\right) q_{1} q_{3}+\left(1+z^{2}\right) q_{3}^{2}\right)}{128 F^{6} \pi\left(-1+z^{2}\right)^{2} q_{1}^{2}}, \\
S_{3}= & \frac{A\left(q_{3}\right) g_{A}^{4}\left(\left(1+z^{2}\right) q_{1}+2 z q_{3}\right)}{128 F^{6} \pi\left(-1+z^{2}\right)^{2} q_{1}}+\frac{A\left(q_{1}\right) g_{A}^{4}\left(2 z q_{1}+\left(1+z^{2}\right) q_{3}\right)}{128 F^{6} \pi\left(-1+z^{2}\right)^{2} q_{3}}+\frac{z A\left(q_{2}\right) g_{A}^{4}\left(-2 q_{1}^{2}+z\left(-5+z^{2}\right) q_{1} q_{3}-2 q_{3}^{2}\right)}{128 F^{6} \pi\left(-1+z^{2}\right)^{2} q_{1} q_{3}}- \\
& \frac{I\left(4: 0,-q_{1}, q_{3} ; 0\right) g_{A}^{4}\left(-4\left(-1+z^{2}\right) M_{\pi}^{2}+\left(1+z^{2}\right) q_{1}^{2}+z\left(3+z^{2}\right) q_{1} q_{3}+\left(1+z^{2}\right) q_{3}^{2}\right)}{32 F^{6}\left(-1+z^{2}\right)^{2}}, \\
S_{4}= & \frac{z A\left(q_{1}\right) g_{A}^{4}\left(\left(1+z^{2}\right) q_{1}+2 z q_{3}\right)}{128 F^{6} \pi\left(-1+z^{2}\right)^{2} q_{3}}+\frac{z A\left(q_{3}\right) g_{A}^{4}\left(2 z q_{1}+\left(1+z^{2}\right) q_{3}\right)}{128 F^{6} \pi\left(-1+z^{2}\right)^{2} q_{1}}+ \\
& \frac{I\left(4: 0,-q_{1}, q_{3} ; 0\right) z g_{A}^{4}\left(4 z\left(-1+z^{2}\right) M_{\pi}^{2}-2 z q_{1}^{2}-\left(1+3 z^{2}\right) q_{1} q_{3}-2 z q_{3}^{2}\right)}{32 F^{6}\left(-1+z^{2}\right)^{2}}- \\
& \frac{z A\left(q_{2}\right) g_{A}^{4}\left(\left(1+z^{2}\right) q_{1}^{2}+z\left(3+z^{2}\right) q_{1} q_{3}+\left(1+z^{2}\right) q_{3}^{2}\right)}{128 F^{6} \pi\left(-1+z^{2}\right)^{2} q_{1} q_{3}}
\end{aligned}
$$




$$
\begin{aligned}
S_{5}= & -\frac{A\left(q_{1}\right) g_{A}^{4} q_{1}\left(\left(1+z^{2}\right) q_{1}+2 z q_{3}\right)}{128 F^{6} \pi\left(-1+z^{2}\right)^{2} q_{3}^{2}}-\frac{A\left(q_{3}\right) g_{A}^{4}\left(2 z q_{1}+\left(1+z^{2}\right) q_{3}\right)}{128 F^{6} \pi\left(-1+z^{2}\right)^{2} q_{3}}+ \\
& \frac{I\left(4: 0,-q_{1}, q_{3} ; 0\right) g_{A}^{4} q_{1}\left(-4 z\left(-1+z^{2}\right) M_{\pi}^{2}+2 z q_{1}^{2}+\left(1+3 z^{2}\right) q_{1} q_{3}+2 z q_{3}^{2}\right)}{32 F^{6}\left(-1+z^{2}\right)^{2} q_{3}}+ \\
& \frac{A\left(q_{2}\right) g_{A}^{4}\left(\left(1+z^{2}\right) q_{1}^{2}+z\left(3+z^{2}\right) q_{1} q_{3}+\left(1+z^{2}\right) q_{3}^{2}\right)}{128 F^{6} \pi\left(-1+z^{2}\right)^{2} q_{3}^{2}}, \\
S_{6}= & -\frac{A\left(q_{3}\right) g_{A}^{4} q_{3}\left(z q_{1}+q_{3}\right)}{128 F^{6} \pi\left(-1+z^{2}\right)}+\frac{A\left(q_{2}\right) g_{A}^{4}\left(q_{1}^{2}-z\left(-3+z^{2}\right) q_{1} q_{3}+q_{3}^{2}\right)}{128 F^{6} \pi\left(-1+z^{2}\right)}-\frac{A\left(q_{1}\right) g_{A}^{4} q_{1}\left(q_{1}+z q_{3}\right)}{128 F^{6} \pi\left(-1+z^{2}\right)}+ \\
& \frac{I\left(4: 0,-q_{1}, q_{3} ; 0\right) g_{A}^{4} q_{1} q_{3}\left(z q_{1}+q_{3}\right)\left(q_{1}+z q_{3}\right)}{32 F^{6}\left(-1+z^{2}\right)}, \\
S_{7}= & \frac{A\left(q_{1}\right) g_{A}^{4}\left(2 M_{\pi}^{2}+q_{3}^{2}\right)}{256 F^{6} \pi\left(-1+z^{2}\right) q_{3}^{2}}-\frac{A\left(q_{2}\right) g_{A}^{4}\left(z q_{3}^{2}\left(z q_{1}+q_{3}\right)+2 M_{\pi}^{2}\left(q_{1}+z q_{3}\right)\right)}{256 F^{6} \pi\left(-1+z^{2}\right) q_{1} q_{3}^{2}}+\frac{z A\left(q_{3}\right) g_{A}^{4}\left(2 M_{\pi}^{2}+q_{3}^{2}\right)}{256 F^{6} \pi\left(-1+z^{2}\right) q_{1} q_{3}}- \\
& \frac{I\left(4: 0,-q_{1}, q_{3} ; 0\right) g_{A}^{4}\left(z q_{1}+q_{3}\right)\left(2 M_{\pi}^{2}+q_{3}^{2}\right)}{64 F^{6}\left(-1+z^{2}\right) q_{3}} .
\end{aligned}
$$

Examining the above results one observes that the individual terms in the expressions for $R_{i}$ and $S_{i}$ are singular for $z= \pm 1, q_{1}=0$ and/or $q_{3}=0$. These singularities, however, cancel in such a way that the resulting terms in Eqs. (A.1) and (A.6) are finite. In principle, it is possible to obtain a representation for functions $R_{i}$ and $S_{i}$ which is free of at least some of the singularities. In particular, the singularities at $z= \pm 1$ can be avoided if one expresses the results in terms of the functions $J_{1}$ and $J_{2}$ defined as

$$
\begin{aligned}
J_{1}\left(d, \vec{q}_{1}, \vec{q}_{3}\right) & =\frac{1}{1-z^{2}}\left\{J\left(d: \overrightarrow{0},-\vec{q}_{1}, \vec{q}_{3}\right)-\frac{1}{2}(1+z)\left[\frac{J\left(d: \overrightarrow{0}, \vec{q}_{1}\right)}{q_{3}^{2}+q_{1} q_{3}}+\frac{J\left(d: \overrightarrow{0}, \vec{q}_{3}\right)}{q_{1}^{2}+q_{1} q_{3}}-\frac{J\left(d: \overrightarrow{0}, \vec{q}_{1}+\vec{q}_{3}\right)}{q_{1} q_{3}}\right]\right. \\
& \left.-\frac{1}{2}(1-z)\left[\frac{J\left(d: \overrightarrow{0}, \vec{q}_{1}\right)}{q_{3}^{2}-q_{1} q_{3}}+\frac{J\left(d: \overrightarrow{0}, \vec{q}_{3}\right)}{q_{1}^{2}-q_{1} q_{3}}+\frac{J\left(d: \overrightarrow{0}, \vec{q}_{1}+\vec{q}_{3}\right)}{q_{1} q_{3}}\right]\right\}, \\
J_{2}\left(d, \vec{q}_{1}, \vec{q}_{3}\right) & =\frac{1}{\left(1-z^{2}\right)^{2}}\left\{J\left(d: \overrightarrow{0},-\vec{q}_{1}, \vec{q}_{3}\right)-\frac{1}{4}(1-z)^{2}\left[\frac{J\left(d: \overrightarrow{0}, \vec{q}_{1}\right)}{q_{3}^{2}-q_{1} q_{3}}+\frac{J\left(d: \overrightarrow{0}, \vec{q}_{3}\right)}{q_{1}^{2}-q_{1} q_{3}}+\frac{J\left(d: \overrightarrow{0}, \vec{q}_{1}+\vec{q}_{3}\right)}{q_{1} q_{3}}\right.\right. \\
& +(1+z)\left[-\frac{8 M^{2}-2 q_{1}^{2}+(d-1)\left(q_{1}-q_{3}\right)\left(2 q_{1}-q_{3}\right)}{(d-1) q_{3}\left(q_{1}-q_{3}\right)^{3}} J\left(d: \overrightarrow{0}, \vec{q}_{1}\right)\right. \\
& \left.\left.+\frac{8 M^{2}-2 q_{3}^{2}+(d-1)\left(q_{1}-q_{3}\right)\left(q_{1}-2 q_{3}\right)}{(d-1) q_{1}\left(q_{1}-q_{3}\right)^{3}} J\left(d: \overrightarrow{0}, \vec{q}_{3}\right)+\frac{2\left(4 M^{2}+(d-2)\left(q_{1}-q_{3}\right)^{2}\right)}{(d-1) q_{1} q_{3}\left(q_{1}-q_{3}\right)^{2}} J\left(d: \overrightarrow{0}, \vec{q}_{1}+\vec{q}_{3}\right)\right]\right] \\
& -\frac{1}{4}(1+z)^{2}\left[\frac{J\left(d: \overrightarrow{0}, \vec{q}_{1}\right)}{q_{3}^{2}+q_{1} q_{3}}+\frac{J\left(d: \overrightarrow{0}, \vec{q}_{3}\right)}{q_{1}^{2}+q_{1} q_{3}}-\frac{J\left(d: \overrightarrow{0}, \vec{q}_{1}+\vec{q}_{3}\right)}{q_{1} q_{3}}\right. \\
& +(1-z)\left[\frac{8 M^{2}-2 q_{1}^{2}+(d-1)\left(q_{1}+q_{3}\right)\left(2 q_{1}+q_{3}\right)}{(d-1) q_{3}\left(q_{1}+q_{3}\right)^{3}} J\left(d: \overrightarrow{0}, \vec{q}_{1}\right)\right. \\
& \left.\left.\left.+\frac{8 M^{2}-2 q_{3}^{2}+(d-1)\left(q_{1}+q_{3}\right)\left(q_{1}+2 q_{3}\right)}{(d-1) q_{1}\left(q_{1}+q_{3}\right)^{3}} J\left(d: \overrightarrow{0}, \vec{q}_{3}\right)-\frac{2\left(4 M^{2}+(d-2)\left(q_{1}+q_{3}\right)^{2}\right)}{(d-1) q_{1} q_{3}\left(q_{1}+q_{3}\right)^{2}} J\left(d: \overrightarrow{0}, \vec{q}_{1}+\vec{q}_{3}\right)\right]\right]\right\},
\end{aligned}
$$

rather than the three-point function $J\left(d: \overrightarrow{0},-\vec{q}_{1}, \vec{q}_{3}\right)$ and uses certain linear combinations of two-point functions and tadpole integrals. In the above expressions, the two-point function is defined as

$$
J\left(d: \vec{p}_{1}, \vec{p}_{2}\right)=\int \frac{d^{d} l}{(2 \pi)^{d}} \frac{1}{\left(\vec{l}+\vec{p}_{1}\right)^{2}+M_{\pi}^{2}} \frac{1}{\left(\vec{l}+\vec{p}_{2}\right)^{2}+M_{\pi}^{2}} .
$$

[1] N. Kalantar-Nayestanaki and E. Epelbaum, Nucl. Phys. News 17, 22 (2007) arXiv:nucl-th/0703089.

[2] D. R. Entem and R. Machleidt, Phys. Rev. C 68, 041001 (2003) arXiv:nucl-th/0304018. 
[3] E. Epelbaum, W. Glöckle and U.-G. Meißner, Nucl. Phys. A 747, 362 (2005) arXiv:nucl-th/0405048.

[4] S. Weinberg, Phys. Lett. B 251, 288 (1990).

[5] S. Weinberg, Nucl. Phys. B 363, 3 (1991).

[6] U. van Kolck, Phys. Rev. C 49, 2932 (1994).

[7] J. L. Friar, D. Huber and U. van Kolck, Phys. Rev. C 59, 53 (1999) arXiv:nucl-th/9809065.

[8] E. Epelbaum, A. Nogga, W. Glöckle, H. Kamada, U.-G. Meißner and H. Witala, Phys. Rev. C 66, 064001 (2002) arXiv:nucl-th/0208023.

[9] E. Epelbaum, Prog. Part. Nucl. Phys. 57, 654 (2006) arXiv:nucl-th/0509032.

[10] A. Nogga, P. Navratil, B. R. Barrett and J. P. Vary, Phys. Rev. C 73, 064002 (2006) arXiv:nucl-th/0511082.

[11] P. Navratil, V. G. Gueorguiev, J. P. Vary, W. E. Ormand and A. Nogga, Phys. Rev. Lett. 99, 042501 (2007) arXiv:nucl-th/0701038.

[12] J. Ley et al., Phys. Rev. C 73, 064001 (2006).

[13] Y. Koike and J. Haidenbauer, Nucl. Phys. A 463, 365 (1987).

[14] H. Witała, D. Hüber and W. Glöckle, Phys. Rev. C 49 R14, (1999).

[15] S. Ishikawa and M. R. Robilotta, Phys. Rev. C 76, 014006 (2007) arXiv:0704.0711 [nucl-th]].

[16] S. C. Pieper, V. R. Pandharipande, R. B. Wiringa and J. Carlson, Phys. Rev. C 64, 014001 (2001) arXiv:nucl-th/0102004.

[17] S. A. Coon and J. L. Friar, Phys. Rev. C 34, 1060 (1986).

[18] M. R. Robilotta, Phys. Rev. C 74, 044002 (2006) [Erratum-ibid. C 74, 059902 (2006)] arXiv:nucl-th/0610046].

[19] N. Fettes, U.-G. Meißner and S. Steininger, Nucl. Phys. A 640, 199 (1998) arXiv:hep-ph/9803266.

[20] V. Bernard, Prog. Part. Nucl. Phys. 60, 82 (2007) arXiv:0706.0312 [hep-ph]].

[21] E. Epelbaum, U.-G. Meißner and W. Glöckle, Nucl. Phys. A 714, 535 (2003) arXiv:nucl-th/0207089.

[22] E. Epelbaum, Phys. Lett. B 639, 456 (2006).

[23] E. Epelbaum, Eur. Phys. J. A 34, 197 (2007) arXiv:0710.4250 [nucl-th]].

[24] V. Bernard, N. Kaiser and U.-G. Meißner, Nucl. Phys. A 615, 483 (1997) arXiv:hep-ph/9611253. 